\title{
Closed-Form Expressions of Electric and Magnetic Near-Fields for the Calibration of Near-Field Probes
}

\author{
A. Boyer, N. Nolhier, F. Caignet and S. Ben Dhia
}

\begin{abstract}
Near-field scan is a powerful method to diagnose EMC issues. Some of related applications require quantitative measurement of near-field, which relies on calibrated near-field probes. A typical and convenient structure to calibrate them is the microstrip line. Although this structure seems simple, determining near-field distribution is not straightforward and 3D electromagnetic simulation is usually preferred. Because of the complexity of electromagnetic solvers and the dependence of results to their configuration which requires a solid expertise, this approach introduces an additional difficulty in the calibration process and a source of uncertainty. This paper proposes closedform expressions based on quasi-static approximation to calculate near-field distribution over microstrip line and simple calibration structures. The paper specifies the frequency limits of this method, which can reach several GHz, covering most of the needs in EMC diagnosis at PCB and IC level. Several probes are calibrated with these formulations, which are validated through the comparison of the near-field measurements above the same case study. A special care is also provided during the calibration process to ensure that the probe characteristics remains independent of the probe height.
\end{abstract}

Index Terms - Near-field scan, near-field probe, calibration, validation, closed-form expressions

\section{INTRODUCTION}

$\mathrm{N}$ EAR-field scan (NFS) is a helpful method to diagnose EMC problems at printed circuit board (PCB) and integrated circuit (IC) levels. Several NFS-based applications, such as near-field to far-field transform for radiated emission estimation [1], contactless current measurement [2] [3], nearfield coupling evaluation [4] or characterization of near-field injection probes [5] require calibrated near-field probes (NFP) in order to quantify electric and magnetic-fields. An important preliminary step in near-field scanning is the probe calibration. It consists in extracting the response of the NFP to the incoming field, in order to reconstruct the actual field distribution. In its simplest or standard form, the calibration process consists in extracting the sensitivity of the probe, assuming the probe has an infinitesimal small size [6]. The probe sensitivity is given by a figure called performance factor $(\mathrm{PF})$, which is the ratio between the induced voltage at the probe terminal and the

This work is supported by the Nanoscan project (CNES $N^{\circ}$ 200111/00) sponsored by Centre National d'Etudes Spatiales (CNES). incoming $\mathrm{E}$ or $\mathrm{H}$-field on the probe. In a more advanced form, the average effect of the field over the probe surface can also be extracted. This type of calibration relies on plane wave spectrum expansion, based on a reference field distribution [7] [8] or on probe transmitting characteristics and reciprocity theorem [9]. It has been successfully applied on single electric or magnetic field probes and on NFP arrays [10]. Except if high resolution scanning is required, in most practical applications, the standard calibration process is sufficient so that this paper focuses only on this calibration process.

Calibration of NFP relies on the measurement of the probe response above a known structure, which produces $\mathrm{E}$ and $\mathrm{H}$ fields that can be determined precisely. Two typical methods propose to use waveguide (e.g. TEM cell) [11] and Helmholtz coils [12] (only for $\mathrm{H}$-field probe) to generate a controlled homogeneous field. However, above PCB and IC, the fields are not homogeneous. They decay rapidly and non-linearly with the distance. The actual measurement height is not known without a proper characterization and both previous methods fail to extract this characteristic. Moreover, calibration of E-field probe in TEM cells is not precise because of the important contribution of the NFP body to the E-field coupling.

Another widely-used method adopted by many authors (e.g. [6] [13] [14] [15]) relies on a transmission line designed on a planar substrate. The microstrip line constitutes a simple structure and a convenient choice up to several tens of $\mathrm{GHz}$, until the quasi-TEM propagation assumption is not valid anymore and other types of structures such as coplanar waveguide [16] should be used. However, microstrip line is sufficient to cover most of the needs of EMC diagnosis of electronic applications (e.g. noise produced by switching-mode power switch, microcontroller, RF platform). This solution is also preconized by standards such as [17] [18]. Although this calibration procedure is well-known and simple, one issue may limit its use by EMC engineers and is related to the evaluation of the field produced by the calibration structure. It is not straightforward due to the inhomogeneous medium. Most of the authors of technical publications about NFP calibration, such as [6]-[10] and [12]-[15], use 3D electromagnetic (EM) simulation. In spite of the great improvement of EM simulators these last years, it requires a solid expertise to select the

A. Boyer, N. Nolhier, F. Caignet and S. Ben-Dhia are with LAAS, Univ. de Toulouse, INSA, Toulouse, France (e-mail: alexandre.boyer@laas.fr, nolhier@1aas.fr, fcaignet@laas.fr, sonia.bendhia@insa-toulouse.fr). 
appropriate solvers and the adequate configuration to ensure the validity and the convergence of the results. Although closedform expressions of near $\mathrm{E}$ and $\mathrm{H}$-fields could provide a practical solution to this issue, few publications address this point. Moreover, closed-form expressions could provide a convenient way to ensure the validity of electromagnetic simulation results. [18] and [19] provide a simple expression to compute the tangential $\mathrm{H}$-field and normal E-field above a microstrip line, but only based on the assumption of an infinitely long thin wire. The effects of the line geometry and dielectric substrate are not considered.

This paper presents several closed-form expressions to compute near $\mathrm{E}$ and $\mathrm{H}$-fields produced by microstrip line and, thus, facilitate the calibration process of NFP. These expressions rely on the quasi-static approximation. As NFP are characterized only in the very-close reactive region of calibration structure (typical scan altitude ranging from 0.5 to $10 \mathrm{~mm}$ ), this assumption remains valid up to several $\mathrm{GHz}$. The paper also addresses the frequency limit of this approach and the impact of parasitic effects such as board edge and substrate losses on the accuracy of closed-form expressions. Moreover, the paper also presents an alternative to the typical set-up used to calibrate NFP at a constant height above microstrip line. The presented methodology proposes to calibrate the NFP at different height above the probe to ensure that the probe characteristics ( $\mathrm{PF}$ and effective height) remain independent of the scan height. This verification is essential for a practical use of near-field scan above PCB, where the distance between NFP and devices under test is not constant.

The paper is organized as follows: Section II describes the calibration process. Then, Section III presents the closed-form expressions of the $\mathrm{E}$ and $\mathrm{H}$ near-fields produced by a microstrip line and specifies the validity range. They are compared with 3D EM simulation results in Section IV. The calibration procedures based on these closed-form expressions is applied on several types of NFP. The calibration results, i.e. the PF and the effective height of the NFP, are shown in Section V. The independence of the PF to the scan altitude and calibration structures is underlined. Finally, Section VI presents the validation case and compares the NF emission map measured by the different NF probes.

\section{CALIBRATION PROCESS}

The general test set-up to calibrate NFP above a planar structure designed on a PCB (e.g. a microstrip line) is described in Fig. 1. The probe is placed precisely by a near-field scanner at a controlled position $\left(\mathrm{x}_{\mathrm{P}}, \mathrm{y}_{\mathrm{P}}\right)$ and a scan altitude $R$ above the calibration structure, which is the distance between the surface of the calibration PCB and the extremity of the probe. The calibration consists in measuring the transfer function between the calibration structure and the probe to be calibrated. It could be done using a spectrum analyzer coupled to a tracking generator or vector network analyzer (VNA). The effect of cables has to be compensated (e.g. by calibrating VNA to the probe and calibration structure inputs). Then, the sensitivity of the probe is given in terms of a performance factor (PF) which is the ratio between the voltage measured across the probe and the incoming field to be measured at the point $\mathrm{P}\left(x_{0} ; y_{0}, r\right)$ placed in the vicinity of the calibration structure. The field is supposed to be calculable by a closed-form expression or a numerical simulation. The $\mathrm{PF}$ for $\mathrm{E}$ and $\mathrm{H}$-field probes are given by (1) and (2), where $V_{2}$ is the voltage measured at port 2 of the VNA, $V_{\text {forw }}$ the voltage of the forward wave produced at port 1 and $\mathrm{r}$ the actual distance of the measurement probe from the calibration structure. $\mathrm{E}$ and $\mathrm{H}$ fields produced by the calibration structure are proportional to $V_{\text {forw }}$. Although all these quantities are complex, only the magnitude will be considered in the next section. E-field probe calibration can be affected by commonmode resonances due to field coupling on the outer shielding of the probe. To attenuate them, ferrite beads can be added around the cables between the probe to the VNA, and between the VNA and the calibration structure.

$$
\begin{aligned}
& P F_{E}(\omega)=\frac{V_{2}(\omega)}{E\left(x_{0}, y_{0}, r, \omega\right)}=\frac{V_{\text {forw }} S_{12}(\omega)}{E\left(x_{0}, y_{0}, r, \omega\right)} \\
& P F_{H}(\omega)=\frac{V_{2}(\omega)}{H\left(x_{0}, y_{0}, r, \omega\right)}=\frac{V_{f o r w} s_{12}(\omega)}{H\left(x_{0}, y_{0}, r, \omega\right)}
\end{aligned}
$$

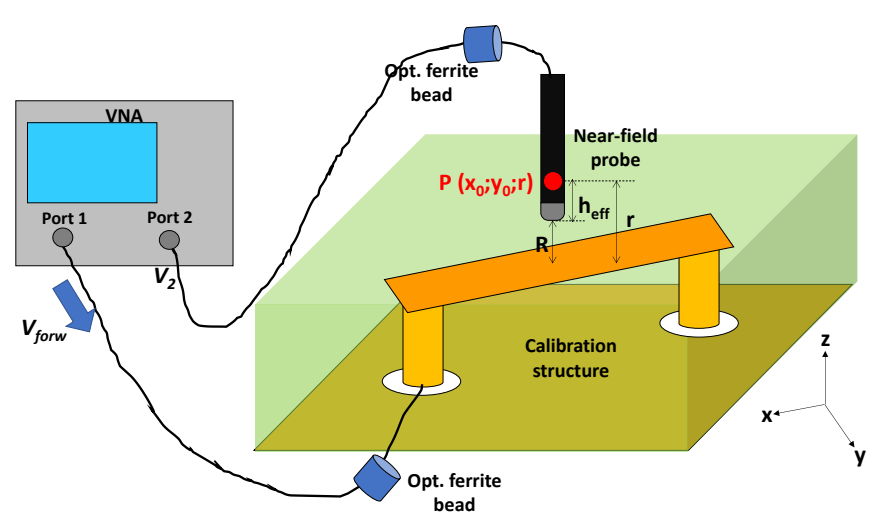

Fig. 1. Typical calibration set-up of near-field probe above calibration structure designed on a PCB

The practical use of a calibrated NFP requires that PF does not depend on the PCB geometry and material. This assumption must be verified during the calibration process, for example with different calibration structures. Moreover, the extracted PF must not depend on the scan altitude $\mathrm{R}$, as if the probe captures the field at one position: $\mathrm{P}\left(\mathrm{x}_{0}, \mathrm{y}_{0}, \mathrm{r}\right)$. However, because of the non-negligible size or the probe and non-homogeneity of the near-field in close proximity of its source, the actual measurement height $r$ is unknown. Thus, the calibration process must also provide the effective measurement height $h_{\text {eff }}$ of the probe as defined by (3). This term is the difference between the probe tip and the point where an ideal infinitesimal probe would provide the same result. In typical calibration done on microstrip line, such as in [15], the effective height is extracted from a measurement at a constant altitude above the line which is compared to simulated field profile at several constant height. The effective height is determined experimentally to ensure the best fit between measured and simulated field profile vs. measurement height $r$.

$$
r=R+h_{e f f}
$$

One drawback of this approach is that the independence of the effective height according to the scan altitude is not verified. 
In this paper, we propose to measure the coupling between the NFP and the microstrip line at different altitude above the line center. The theoretical field is computed at different measurement height and the PF is determined according to (1) or (2). $h_{\text {eff }}$ is finally adjusted to ensure a PF as constant as possible according to the scan altitude $R$. Having several calibration structures with different geometries, substrate thickness and material can be judicious to ensure that the PF and effective height are not dependent on the calibration structure.

An adequate calibration structure must be simple enough and symmetrical to facilitate the computation of $\mathrm{E}$ and $\mathrm{H}$ fields. Moreover, the voltage and the current must be precisely known over the calibration frequency range, as they constitute the sources of near-field emission. The electrical parameters of the calibration structure (characteristic impedance and propagation constant) must be characterized properly to evaluate voltage and current along the structure. Thus, line losses and dispersion must remain limited to guarantee a precise knowledge on line parameters, current and voltage on the calibration structure. The practical use of the microstrip line as a calibration structure can be limited by the dispersion, which remains negligible if the frequency is less than the limit $F_{\text {disp }}$ given by (4), where $Z_{C}$ is the characteristic impedance of the line and $h$ the substrate thickness in cm [20].

$$
F_{\text {disp }}(G H z)=0.3 \sqrt{\frac{Z_{c}}{h \sqrt{\varepsilon_{r^{-1}}}}}
$$

For a microstrip line, the characteristic impedance has to be controlled to be able to limit current and voltage variations along the line. The line should also be designed symmetrically and far from board edges to reduce the impact of edge radiation. Finally, low-loss high frequency substrate is advised to limit the dispersion of line characteristics.

\section{CloSED-Form EXPRESSIONS OF THE NEAR-FIELDS ABOVE MICROSTRIP LINE}

The following sections describe the equations used to determine the $\mathrm{E}$ and $\mathrm{H}$-fields produced by a microstrip line in near-field region, in order to extract the PF. To obtain simple closed-form expressions, two assumptions have to be made: quasi-static conditions are assumed and the probe is placed close enough to consider that the reactive part of the field dominates. Obviously, these assumptions are valid only in low frequency and short distance, so that we have to define the validity range in space and frequency domains.

Let us consider any structure with a known arbitrary charge $\rho$ and current density $J$ distribution on any point $r^{\prime}$. The electric and magnetic fields on any point $r_{P}$ are given by Jefimenko equations, as shown by (5) and (6) where $t_{r}$ is the retarded time. Here, the main interest of these equations is not the derivation of closed-form expressions of $\mathrm{E}$ and $\mathrm{H}$ fields because it cannot lead in integrable expressions. However, they clarify the sources of electromagnetic fields and help to determine limits of the reactive region.

$$
\begin{gathered}
E(r, t)=\frac{1}{4 \pi \varepsilon_{0}} \int\left[\left(\frac{\rho\left(r^{\prime}, t_{r}\right)}{\left|r_{p}-r^{\prime}\right|^{3}}+\frac{1}{\left|r_{p}-r_{\prime}\right|^{2} c} \frac{d \rho\left(r^{\prime}, t_{r}\right)}{d t}\right) \times\left(r_{P}-r^{\prime}\right)-\right. \\
\left.\frac{1}{\left|r_{p}-r^{\prime}\right| c^{2}} \frac{d J\left(r^{\prime}, t_{r}\right)}{d t}\right] d^{3} r^{\prime}
\end{gathered}
$$

$H(r, t)=\frac{1}{4 \pi} \int\left(\frac{J\left(r^{\prime}, t_{r}\right)}{\left|r_{p}-r^{\prime}\right|^{3}}+\frac{1}{\left|r_{p}-r^{\prime}\right|^{2} c} \frac{d J\left(r^{\prime}, t_{r}\right)}{d t}\right) \times\left(r_{P}-r^{\prime}\right) d^{3} r^{\prime}$

Both equations indicate that current and charge distribution constitute the source of $\mathrm{E}$ and $\mathrm{H}$ fields. In (5) and (6), the first term is the reactive field, which is directly related to the charge or current distribution and not to their time derivative. The second term in (6) and the third term in (5) are related to the radiated contribution, while the second term in (5) is related to induction effect. In close near-field region, the reactive contribution dominates if the first term of (5) and (6) is larger than the others. Let us suppose that the structure is excited by a sine wave source. The first term is larger than the second one if the condition (7) is met, where $\lambda_{0}$ is the wavelength in air.

$$
\left|r_{P}-r^{\prime}\right|<\frac{\lambda_{0}}{2 \pi}
$$

Let us suppose that the propagation speed along the considered structure is $v$ and $\beta$ is the phase constant. According to the continuity equation (8) in harmonic regime, the first term in (5) is larger than the third one if (9) is met. According to (7) and (9), if (7) is met, only the reactive terms dominates and (5) and (6) can be simplified to the well-known Gauss theorem and Biot and Savart law. If the structure under test is electrically small, quasi-static approximation can be applied and a first frequency limit (10) can be defined to ensure the reactive region assumption, according to the measurement distance $r$. The evolution of this frequency limit according to the measurement height is plotted in Fig. 5. For typical NFS measurement height (e.g. 1-10 mm), (4) is verified between 5 and $50 \mathrm{GHz}$.

$$
\begin{gathered}
\frac{d \rho\left(r^{\prime}, t_{r}\right)}{d t}=-\nabla J\left(r^{\prime}, t_{r}\right) \rightarrow J=\frac{\omega}{\beta} \rho=v \rho \\
\left|r_{P}-r^{\prime}\right|<\frac{\lambda_{0}}{2 \pi} \frac{c}{v} \\
F_{\max }<\frac{c}{2 \pi r_{p}}
\end{gathered}
$$

However, for typical microstrip line, quasi-static approximation cannot be verified along all the line up to the frequency limit in (10). Nevertheless, E and H fields decrease very rapidly with distance in near-field region so that they depend mainly on the charge and current distribution along the portion of the microstrip line placed just below the measurement point. $\mathrm{E}$ and $\mathrm{H}$ fields can be determined according to Gauss theorem (11) and Biot and Savart law (12) if this portion is electrically small. The evaluation of the length of this portion will be addressed later in part $\mathrm{C}$, once closed-form expressions of $\mathrm{E}$ and $\mathrm{H}$ fields will be extracted.

$$
\begin{aligned}
& E(r)=\frac{1}{4 \pi \varepsilon_{0}} \int \frac{\rho\left(r^{\prime}\right)}{\left|r_{p}-r^{\prime}\right|^{3}}\left(r_{p}-r^{\prime}\right) d^{3} r^{\prime} \\
& H(r)=\frac{1}{4 \pi} \int \frac{J\left(r^{\prime}\right)}{\left|r_{p}-r^{\prime}\right|^{3}} \times\left(r_{p}-r^{\prime}\right) d^{3} r^{\prime}
\end{aligned}
$$




\section{A. Expression of Near H-Field above the microstrip line}

\section{1) Tangential H-field above the microstrip line}

Let us consider the microstrip line described in Fig. 2, whose characteristic impedance is $Z_{C}$ and propagation constant is $\gamma$. The origin $\mathrm{O}$ of the Cartesian coordinate system for the analysis of the microstrip line is placed at the center of the line. The effect of board edges is neglected and the ground plane is supposed infinitely large. Moreover, substrate losses are neglected, so that the contribution of the ground plane can be accounted according to image theory. The field captured by the probe depends mainly on the current flowing along the line portion placed below the probe. If this portion is electrically small, the current $I$ is supposed constant along it. (2) can be rewritten to (13) to compute the performance factor, where $F_{c a l}^{H}$ is the $\mathrm{H}$-field produced by a calibration structure $c a l$ when it is excited by a normalized excitation. It depends on the line geometry and the measurement point position $\mathrm{P}$ which related the current on $\mathrm{O}$ and the $\mathrm{H}$ field. $P F_{H}$ depends also on the actual measurement height $r$, which is linked to the scan altitude $R$ and the probe effective height $h_{\text {eff }}$ according to (3).

$$
P F_{H}(f)=\frac{V_{\text {forw }} S_{12}(f)}{I\left(f, x_{0}\right) F_{c a l}^{H}\left(x_{0}, y_{0}, r\right)}
$$

If $\mathrm{P}$ is placed at a distance $r$ above the center of the microstrip line $\left(x_{0}=-L / 2\right.$ and $\left.y_{0}=0\right)$, the expression of the current is given by (14), where $Z_{0}$ is the characteristic impedance of the VNA. In case of a perfect matching of the microstrip line, the expression simplifies to (15). A preliminary S-parameter characterization of the microstrip line and termination load is recommended to determine the current accurately.

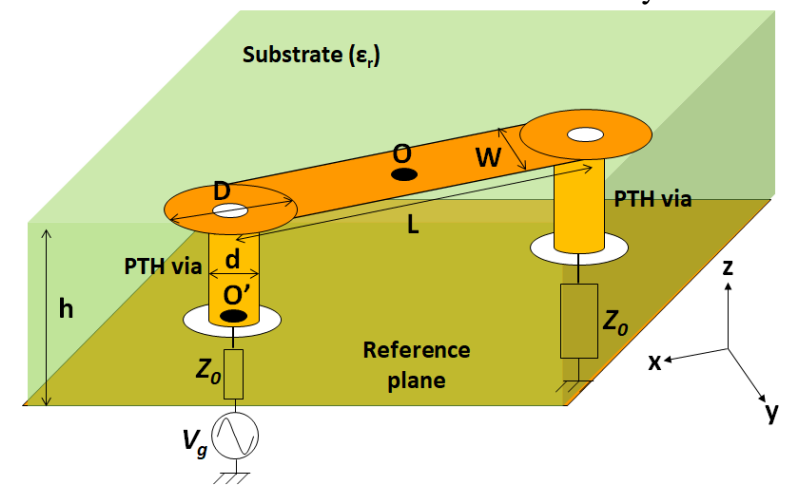

Fig. 2. Geometry of the calibration microstrip line

$$
\begin{gathered}
I\left(f, \frac{L}{2}\right)=2 \frac{Z_{0}+Z_{C}-\left(Z_{0}-Z_{C}\right) e^{-j \gamma L}}{\left(Z_{0}+Z_{C}\right)^{2}-\left(Z_{0}-Z_{C}\right)^{2} e^{-j 2 \gamma L}} e^{-j \gamma \frac{L}{2}} V_{\text {forw }} \\
I\left(f, \frac{L}{2}\right)=\frac{V_{\text {forw }}}{Z_{0}} e^{-j \gamma \frac{L}{2}}
\end{gathered}
$$

If the line thickness is neglected, the tangential $\mathrm{H}$-field at any point $\left(x_{P}, y_{P}, r\right)$ can be determined by considering a uniform current density $J$ flowing through a rectangular conductive sheet. If the current is oriented along $\mathrm{x}$, the tangential $\mathrm{H}$-field produced by the current distribution along the trace can be determined from the resolution of integral (12). According to (16) to (19), (12) is rewritten in (20). After solving this integral, the contribution of the geometry to the H-field in $(\mathrm{P})$ is given by (21). If the measurement point is placed above the center of the line $\mathrm{O}$, this equation simplifies to (22). The presence of the ground plane can be considered by the method of images and the tangential $\mathrm{H}$-field is given by (23). $\mathrm{PF}$ of tangential $\mathrm{H}$-field probe based on a microstrip line is extracted from (13) where $F_{c a l}^{H}$ is replaced by $F_{M S}^{H y}$.

$$
\begin{gathered}
r_{p}=\left(x_{p}, y_{p}, r\right) \quad r^{\prime}=(x, y, 0) \\
\left|r_{p}-r^{\prime}\right|=\sqrt{r^{2}+\left(x-x_{P}\right)^{2}+\left(y-y_{P}\right)^{2}} \\
J\left(r^{\prime}\right) \times\left(r_{p}-r^{\prime}\right)=\left(0, J r, J\left(y-y_{P}\right)\right) \\
H_{Y}=\frac{J}{4 \pi} \int_{-L / 2}^{+L / 2} \int_{-W / 2}^{+W / 2} \frac{r}{\left(r^{2}+\left(x-x_{P}\right)^{2}+\left(y-y_{P}\right)^{2}\right)^{3 / 2}} d y d x
\end{gathered}
$$

$$
\begin{aligned}
& F_{M S}^{H y}=\frac{1}{4 \pi w}\left[\operatorname{atan}\left(\frac{\left(\frac{w}{2}-x_{P}\right)\left(\frac{L}{2}-y\right)}{r \sqrt{r^{2}+\left(\frac{w}{2}-x_{P}\right)^{2}+\left(\frac{L}{2}-y_{P}\right)^{2}}}\right)+\right. \\
& \operatorname{atan}\left(\frac{\left(\frac{w}{2}+x_{P}\right)\left(\frac{L}{2}-y\right)}{r \sqrt{r^{2}+\left(\frac{w}{2}+x_{P}\right)^{2}+\left(\frac{L}{2}-y_{P}\right)^{2}}}\right)+\operatorname{atan}\left(\frac{\left(\frac{w}{2}-x_{P}\right)\left(\frac{L}{2}+y\right)}{r \sqrt{r^{2}+\left(\frac{w}{2}-x_{P}\right)^{2}+\left(\frac{L}{2}+y_{P}\right)^{2}}}\right)+ \\
& \left.\operatorname{atan}\left(\frac{\left(\frac{w}{2}+x_{P}\right)\left(\frac{L}{2}+y\right)}{r \sqrt{r^{2}+\left(\frac{w}{2}+x_{P}\right)^{2}+\left(\frac{L}{2}+y_{P}\right)^{2}}}\right)\right] \\
& F_{M S}^{H y}=\frac{1}{\pi w}\left[\operatorname{atan}\left(\frac{F_{M S}^{H y}=\frac{1}{\pi w}}{4 r \sqrt{r^{2}+\left(\frac{w}{2}\right)^{2}+\left(\frac{L}{2}\right)^{2}}}\right)-\operatorname{atan}\left(\frac{w L}{4 r \sqrt{r^{2}+\left(\frac{w}{2}\right)^{2}+\left(\frac{L}{2}\right)^{2}}}\right)\right.
\end{aligned}
$$

\section{2) Influence of PTH via on the tangential $\mathrm{H}$-field}

In the microstrip line shown in Figs. 1 and 2, both ends are terminated by RF connectors mounted on the opposite face of the trace. The central pins are considered as plated-hole (PTH) vias. Edge-mounted RF connectors are an alternative where no termination PTH vias exist, but it is not considered here. Except if the microstrip line is infinitely long, the influence of the both PTH vias on the tangential $\mathrm{H}$-field cannot be neglected. As the radius of a typical PTH via is about some hundreds of $\mu \mathrm{m}$, they can be considered as a thin vertical wire. Due to the small thickness of PCB (e.g. $1.6 \mathrm{~mm}$ ), PTH via length remains electrically small up to nearly $10 \mathrm{GHz}$. For the microstrip line structure, the via is a h-long vertical wire which crosses a large ground plane. According to (12) and (16)-(19) and the image theory, the tangential H-field created by a PTH via is given by (24). To derive this result, the origin of the cartesian coordinate system has been shifted in O', as shown in Fig. 2. If the measurement point is placed above the center of the line $\mathrm{O}$ and if the second via is accounted for, this equation simplifies and the contribution of vias to the tangential $\mathrm{H}$-field is given by (25). $F_{v i a}^{H y}$ and $F_{M S}^{H y}$ can be added and replace $F_{c a l}^{H}$ in (13) to extract the $\mathrm{PF}$ of tangential $\mathrm{H}$-field from the microstrip line calibration structure. 


$$
\begin{gathered}
H_{Y}=\frac{I}{4 \pi} \int_{-h}^{h} \frac{1}{\left(x_{P}^{2}+y_{P}^{2}+\left(z-z_{P}\right)^{2}\right)^{3 / 2}} d z \\
=\frac{I}{4 \pi\left(x_{P}^{2}+y_{P}^{2}\right)}\left(\frac{z_{P}+h}{\sqrt{x_{P}^{2}+y_{P}^{2}+\left(z_{P}+h\right)^{2}}}-\frac{z_{P}-h}{\sqrt{x_{P}^{2}+y_{P}^{2}+\left(z_{P}-h\right)^{2}}}\right) \\
F_{\text {via }}^{H y}=\frac{1}{\pi L}\left(\frac{r+2 h}{\sqrt{(r+2 h)^{2}+\left(\frac{L}{2}\right)^{2}}}-\frac{r}{\sqrt{r^{2}+\left(\frac{L}{2}\right)^{2}}}\right)
\end{gathered}
$$

\section{3) Normal H-field above the microstrip line}

The normal $\mathrm{H}$-field produced by the microstrip line is determined according to (12) and (16)-(19). The terminating vias do not contribute to the normal $\mathrm{H}$-field. The normal $\mathrm{H}$-field can be found by solving the integral (26). When solved, PF can be computed according to $F_{M S}^{H z 1}$ (27) which depends on the structure geometry and the measurement point position. If the measurement point is placed above $\mathrm{O},(27)$ simplifies to (30).

$$
\begin{gathered}
H_{Z}=\frac{J}{4 \pi} \int_{-L / 2}^{+L / 2} \int_{-}^{+} W / 2 \frac{y / 2}{\left(r^{2}+\left(x-x_{P}\right)^{2}+\left(y-y_{P}\right)^{2}\right)^{3 / 2}} d y d x \\
F_{M S}^{H Z 1}=\frac{-1}{4 \pi w} \ln \left(\frac{\left(\frac{L}{2}-x_{0}\right)+\sqrt{\left(\frac{L}{2}-x_{0}\right)^{2}+a^{2}}}{-\left(\frac{L}{2}+x_{0}\right)+\sqrt{\left(\frac{L}{2}+x_{0}\right)^{2}+a^{2}}} \times \frac{\left(\frac{L}{2}+x_{0}\right)+\sqrt{\left(\frac{L}{2}+x_{0}\right)^{2}+b^{2}}}{-\left(\frac{L}{2}-x_{0}\right)+\sqrt{\left(\frac{L}{2}-x_{0}\right)^{2}+b^{2}}}\right) \\
a^{2}=r^{2}+\left(\frac{w}{2}+y_{0}\right)^{2} \\
b^{2}=r^{2}+\left(\frac{w}{2}-y_{0}\right)^{2} \\
F_{M S}^{H Z 1}=\frac{-1}{4 \pi w} \ln \left(\frac{\frac{L}{2}+\sqrt{\left(\frac{L}{2}\right)^{2}+a^{2}}}{-\frac{L}{2}+\sqrt{\left(\frac{L}{2}\right)^{2}+a^{2}}} \times \frac{\frac{L}{2}+\sqrt{\left(\frac{L}{2}\right)^{2}+b^{2}}}{-\frac{L}{2}+\sqrt{\left(\frac{L}{2}\right)^{2}+b^{2}}}\right)
\end{gathered}
$$

According to the methods of images, the contribution of the ground plane is given by (31) and the total normal field by (34). $F_{M S}^{H Z}$ can replace $F_{c a l}^{H}$ in (13) to extract the $\mathrm{PF}$ of normal $\mathrm{H}$-field from the microstrip line calibration structure.

$$
\begin{gathered}
F_{M S}^{H z 2}=\frac{1}{4 \pi w} \ln \left(\frac{\frac{L}{2}+\sqrt{\left(\frac{L}{2}\right)^{2}+a^{\prime 2}}}{-\frac{L}{2}+\sqrt{\left(\frac{L}{2}\right)^{2}+a^{\prime 2}}} \times \frac{\frac{L}{2}+\sqrt{\left(\frac{L}{2}\right)^{2}+b^{\prime 2}}}{-\left(\frac{L}{2}\right)+\sqrt{\left(\frac{L}{2}\right)^{2}+b^{2}}}\right) \\
a^{\prime 2}=(r+2 h)^{2}+\left(\frac{w}{2}+y_{0}\right)^{2} \\
b^{\prime 2}=(r+2 h)^{2}+\left(\frac{w}{2}-y_{0}\right)^{2} \\
F_{M S}^{H z}=F_{M S}^{H z 1}+F_{M S}^{H z 2}
\end{gathered}
$$

\section{B. Expression of Near E-Field above Calibration Structure}

The derivation of closed-form expression of E-field in the near-field region of the calibration structure is similar to the approach to determine $\mathrm{H}$-field. The only difference comes from the influence of the grounded substrate, which complicates the extraction of closed-form expressions of the E-field.

\section{1) Effect of the grounded substrate}

Compared to the $\mathrm{H}$-field, the computation of the E-field is more challenging due to the boundaries of the substrate. The air-dielectric interface and the ground plane lead to multiple reflections between them, leading to a complex relationship between the E-field produced by a calibration structure and its geometry. In order to determine a closed-form expression of the E-field in such a non-homogeneous medium, a possible approach is to extract the Green's functions rigorously. Traditionally, the Green's function in spatial domain is represented by the Sommerfeld integrals extracted from the Maxwell equations and boundary conditions at each layer interface. The transform in spectral domain has been widely used in microwave circuits applications in order to obtain closed-form expressions of Green's function in multi-layer problems, followed by a numerical integration of the Sommerfeld integral expressed in spectral domain, requiring Fourier transform [21] [22]. Although it can lead to precise evaluations of field in the near-field region of interconnects designed on multilayer substrate [5], the development of this approach can be time-consuming and complex.

In the case of a two-layer substrate and if losses remain reasonable enough to be considered as negligible, the image theory is a more practical alternative which does not require a complex numerical integration. The effect of the ground plane and the air-dielectric interface can be modeled by a series of multiple virtual and partial images whose distance to the substrate surface and excitation depends on substrate thickness $h$, measurement height $r$ and dielectric permittivity $\varepsilon_{r}$. The method, initially presented in [23], was used to derive characteristic impedance and propagation velocity of microstrip line. It can be reused to determine closed-form expressions of the near E-field surrounding basic interconnects designed on a two-layer lossless substrate. To illustrate it, let us consider a charge distribution $\rho$ located at the substrate surface. The Efield is the sum of the direct contribution of this charge and those of the multiple reflections. They can be modeled by virtual charges placed under the substrate surface, as illustrated in Fig. 3. The two first primary images, $\rho_{I}$ and $\rho_{2}$, are related to the primary images due to one reflection on the air-dielectric interface and the ground plane respectively. The other secondary images, $\rho_{1}{ }^{n}$ and $\rho_{2}{ }^{n}$, are related to the multiple reflections of both primary images on both interfaces.

The contribution of the charge distribution $\rho$ to the E-field in $\mathrm{P}$ is given by a function $F(\rho, r)$ which depends on the structure geometry. The contribution $F^{E}$ of all the partial charge to the Efield can be determined by (35), where the first term is the direct contribution of $\rho$, the second and third terms are the contributions of both primary images and the two last terms are the contributions of the secondary images. $K$, the reflection coefficient of the E-field flux at the air dielectric interface, is given by (36). $\mathrm{K}$ ' is the attenuation provided to the ground plane image due to the substrate. It is the product of the transmission coefficient of the E-field flux at the air-dielectric interface with the one at the dielectric-air interface, as given by (37). The computation of the grounded substrate effect does not require any Fourier transform, only the sum of a finite number of closed-form functions $F(\rho, r)$. For microstrip line designed on typical PCB for electronic products ( $h$ ranging between 0.2 and $2 \mathrm{~mm}, w<3 \mathrm{~mm}, L<10 \mathrm{~cm}, \varepsilon_{r}$ between 2 and 5) and for scan measurement height less than $10 \mathrm{~mm}, 3$ to 6 secondary images are sufficient to converge at less than $10 \%$ of the actual E-field value, and 5 to 8 secondary images to converge at less than $1 \%$ 
of the actual E-field value.

$$
\begin{gathered}
F^{E}=F(\rho, r)+F(K \rho, r)+F\left(-K^{\prime} \rho, r+2 h\right)- \\
\sum_{n=1}^{\infty}\left(F\left(K^{\prime K^{2 n-1}} \rho, r+4 n h\right)-F\left(K^{\prime} K^{2 n} \rho, r+(4 n+2) h\right)\right) \\
K=\frac{1-\varepsilon_{r}}{1+\varepsilon_{r}} \\
K^{\prime}=(1-K)(1+K)=1-K^{2}
\end{gathered}
$$

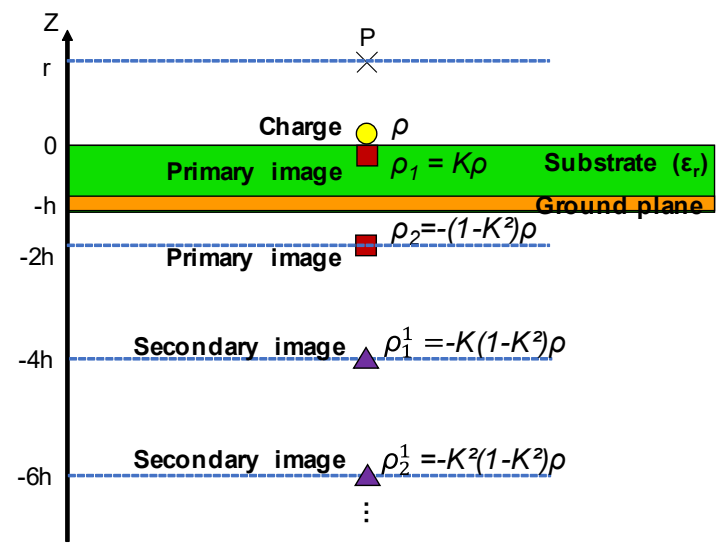

Fig. 3. Multiple partial image model for a charge density placed above a grounded substrate

\section{2) Normal and tangential E-field above the microstrip line}

Let us consider the microstrip line described in Fig. 2. The field captured by the E-field probe depends mainly on the charge distribution on the line portion placed below the probe. If this portion is supposed electrically small, the charge and thus the voltage $V$ is supposed constant along it. (1) can be rewritten to (38) to compute the performance factor, where $F_{M S}^{E}$ is a function which gives the field component $E$ produced by a calibration structure cal when it is excited by a normalized excitation. It depends on the line geometry and the measurement point position P. $P F_{E}$ depends also on the actual measurement height $r$, which is linked to the scan altitude $R$ and the probe effective height $h_{\text {eff }}$ according to (3).

$$
P F_{E}(f)=\frac{V_{f o r w} S_{12}(f)}{V\left(f, x_{0}\right) F_{c a l}^{E}\left(x_{0}, y_{0}, r\right)}
$$

If $\mathrm{P}$ is placed above the center of the microstrip line $\left(x_{0}=-L / 2\right.$ and $y_{0}=0$ ), the voltage along the line is given by (39). If the line is perfectly matched, the expression simplified to (40). A preliminary S-parameter characterization of the microstrip line and termination load is recommended to determine the voltage accurately.

$$
\begin{gathered}
V\left(f, \frac{L}{2}\right)=2 \frac{Z_{0}+Z_{C}+\left(Z_{0}-Z_{C}\right) e^{-j \gamma L}}{\left(Z_{0}+Z_{C}\right)^{2}-\left(Z_{0}-Z_{C}\right)^{2} e^{-j 2 \gamma L}} Z_{C} e^{-j \gamma \frac{L}{2}} V_{\text {forw }} \\
V\left(f, \frac{L}{2}\right)=V_{\text {forw }} e^{-j \gamma \frac{L}{2}}
\end{gathered}
$$

If the line thickness is neglected, the normal E-field at any point $\left(x_{P}, y_{P}, r\right)$ can be determined by considering a uniform charge distribution $\rho$ on a rectangular conductive sheet. For a microstrip line, the charge distribution on any point is related to the voltage and the per-unit-length capacitance $C_{\text {lin }}$, as given by (41). From the Gauss theorem (11) and (16)-(19), the normal E field is computed at any point $\mathrm{P}$ by the integral (42) whose solution is given by (43). If the measurement point is placed above the center of the line $\mathrm{O}$, this equation simplifies to (44). Then, the influence of the grounded substrate is considered by replacing $F^{E}$ by $F_{M S}^{E Z}$ in (35). $F_{M S}^{E Z}$ can replace $F_{c a l}^{E}$ in (38) to extract the PF of normal E-field from the microstrip line calibration structure.

$$
\begin{gathered}
\rho(x)=C_{\text {lin }} V(x) \\
E_{Z}=\frac{\rho}{4 \pi \varepsilon_{0}} \int_{-L / 2}^{+L / 2} \int_{-W / 2}^{+W / 2} \frac{r}{\left(r^{2}+\left(x-x_{P}\right)^{2}+\left(y-y_{P}\right)^{2}\right)^{3 / 2}} d y d x \\
F_{M S}^{E Z}=\frac{C_{\text {lin }}}{4 \pi \varepsilon_{0} w L}\left[\operatorname{atan}\left(\frac{\left(\frac{w}{2}-x_{P}\right)\left(\frac{L}{2}-y\right)}{r \sqrt{r^{2}+\left(\frac{w}{2}-x_{P}\right)^{2}+\left(\frac{L}{2}-y_{P}\right)^{2}}}\right)+\right. \\
\operatorname{atan}\left(\frac{\left(\frac{w}{2}+x_{P}\right)\left(\frac{L}{2}-y\right)}{r \sqrt{r^{2}+\left(\frac{w}{2}+x_{P}\right)^{2}+\left(\frac{L}{2}-y_{P}\right)^{2}}}\right)+\operatorname{atan}\left(\frac{\left(\frac{w}{2}-x_{P}\right)\left(\frac{L}{2}+y\right)}{r \sqrt{r^{2}+\left(\frac{w}{2}-x_{P}\right)^{2}+\left(\frac{L}{2}+y_{P}\right)^{2}}}\right)+ \\
\left.\operatorname{atan}\left(\frac{\left(\frac{w}{2}+x_{P}\right)\left(\frac{L}{2}+y\right)}{r \sqrt{r^{2}+\left(\frac{w}{2}+x_{P}\right)^{2}+\left(\frac{L}{2}+y_{P}\right)^{2}}}\right)\right] \\
F_{M S}^{E Z}=\frac{C_{l i n}}{\pi \varepsilon_{0} w L} \operatorname{atan}\left(\frac{w L}{4 r \sqrt{r^{2}+\left(\frac{w}{2}\right)^{2}+\left(\frac{L}{2}\right)^{2}}}\right)
\end{gathered}
$$

Similarly to (42), the tangential E-field can be computed at any point $\mathrm{P}$ by (45) whose solution is given by (46). If the measurement point is placed above the center of the line $\mathrm{O}$, this equation simplifies to (49). Then, the influence of the grounded substrate is considered by replacing $F$ by $F_{M S}^{E y}$ in (35). $F_{M S}^{E y}$ can replace $F_{c a l}^{E}$ in (34) to extract the PF of tangential E-field from the microstrip line calibration structure.

$$
\begin{gathered}
E_{Y}=\frac{\rho}{4 \pi \varepsilon_{0}} \int_{-L / 2}^{+L / 2} \int_{-W / 2}^{+W / 2} \frac{y-y_{P}}{\left(r^{2}+\left(x-x_{P}\right)^{2}+\left(y-y_{P}\right)^{2}\right)^{3 / 2}} d y d x \\
F_{M S}^{E y}=\frac{C_{l i n}}{4 \pi \varepsilon_{0} L w} \ln \left(\frac{\left(\frac{L}{2}-x_{0}\right)+\sqrt{\left(\frac{L}{2}-x_{0}\right)^{2}+a^{2}}}{-\left(\frac{L}{2}+x_{0}\right)+\sqrt{\left(\frac{L}{2}+x_{0}\right)^{2}+a^{2}}} \times \frac{\left(\frac{L}{2}+x_{0}\right)+\sqrt{\left(\frac{L}{2}+x_{0}\right)^{2}+b^{2}}}{-\left(\frac{L}{2}-x_{0}\right)+\sqrt{\left(\frac{L}{2}-x_{0}\right)^{2}+b^{2}}}\right) \\
a^{2}=r^{2}+\left(\frac{w}{2}+y_{0}\right)^{2} \\
b^{2}=r^{2}+\left(\frac{w}{2}-y_{0}\right)^{2} \\
F_{M S}^{E y}=\frac{C_{l i n}}{4 \pi \varepsilon_{0} L w} \ln \left(\frac{\frac{L}{2}+\sqrt{\left(\frac{L}{2}\right)^{2}+a^{2}}}{-\frac{L}{2}+\sqrt{\left(\frac{L}{2}\right)^{2}+a^{2}}} \times \frac{\frac{L}{2}+\sqrt{\left(\frac{L}{2}\right)^{2}+b^{2}}}{-\frac{L}{2}+\sqrt{\left(\frac{L}{2}\right)^{2}+b^{2}}}\right)
\end{gathered}
$$

\section{3) Influence of PTH via on the normal E-field}

For short microstrip line, the influence of both termination PTH vias on the normal E-field cannot be neglected. Moreover, a single PTH via can constitute a miniature calibration structure. The E-field surrounding the PTH via depends mainly on the charge distribution on the annular ring with a diameter $\mathrm{D}$, which is related to the excitation voltage and the via capacitance $C_{P T H}$. If the charge distribution is supposed homogeneous, the normal E-field at the vertical of the annular ring can be derived from Gauss theorem (11) after cylindrical coordinate transform. The integral (50) in cylindric system coordinates, whose center $\mathrm{O}$ is placed at the center of the top of the via, is obtained and its solution is given by (51). Then, the influence of the grounded substrate is considered by replacing $F$ by $F_{v i a}^{E Z}$ in (35). $F_{v i a}^{E Z}$ and $F_{M S}^{E Z}$ can be added and replace $F_{c a l}^{E}$ in (34) to extract the PF of normal E-field from the microstrip line calibration structure. 


$$
\begin{gathered}
E_{Z}=\frac{C_{P T H} V}{4 \pi \varepsilon_{0}} \int_{0}^{D} / 2 \int_{0}^{2 \pi} \frac{r \rho}{\left(r^{2}+\rho^{2}\right)^{3 / 2}} d \theta d \rho \\
F_{\text {via }}^{E Z}=\frac{2 C_{P T H}}{\pi \varepsilon_{0} D^{2}}\left(1-\frac{r}{\sqrt{r^{2}+\left(\frac{D}{2}\right)^{2}}}\right)
\end{gathered}
$$

\section{Validity range and extension of the quasi-static approximation}

The previous equations assume that the current is nearly constant on the portion of the line placed below the probe. A frequency limit to verify this assumption can be derived from the closed-form expressions of $\mathrm{E}$ and $\mathrm{H}$ fields produced by the microstrip line. It consists in determining the length $\Delta L$ of the line portion that has a major influence on the $\mathrm{H}$-field. As long as this line portion is electrically small, the quasi-static approximation is valid to compute the field produced by the microstrip line. This length does not depend only on the line geometry but also on the measurement height $\mathrm{r}$.

The different formulations of $\mathrm{Hy}, \mathrm{Hz}$ and Ez produced by a normalized excitation ((23), (34) and (44)) can have different expression, but they follow the same evolution according to their parameters. When the field amplitude is plotted according to the line length, its evolution converges according the trend shown in Fig. 4. The relative difference between the Hy fields produced by a microstrip line with a finite length and an infinitely long line is computed. In the following example, the line width is set to $1 \mathrm{~mm}$ and the substrate height to $1.6 \mathrm{~mm}$. The difference is plotted for three measurement heights $r$. After a rapid increase of the field amplitude, it tends to stabilize slowly according to the line length. Whatever $r$, a local maximum is observed for a length $\Delta L(r=x x \mathrm{~mm})$ where $\mathrm{xx}$ refers the $r$ value in $\mathrm{mm}$. It is interesting to note that the relative difference is comprised between 5 and $10 \%$. When the field produced by a long line is considered at a measurement height $\mathrm{r}$, the contribution of the $\Delta L$ long portion of the line below the measurement point is dominant as it contributed to 90 to $95 \%$ of the field amplitude. Whatever the line characteristics and measurement height, the dominant portion of the line can be determined as line length where this local maximum appears. The same behavior is observed for the other expressions of tangential and normal $\mathrm{E}$ and $\mathrm{H}$ fields.

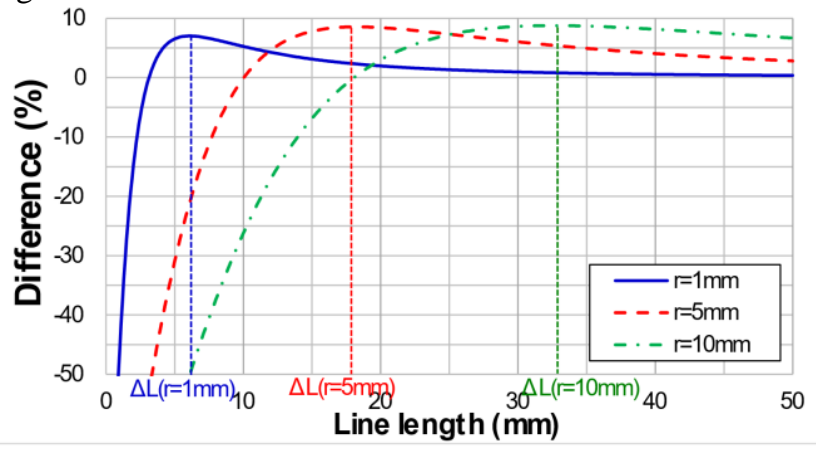

Fig. 4. Relative difference between the Hy field produced by a finite length line and an infinitely long line vs. line length $(\mathrm{w}=1 \mathrm{~mm}, \mathrm{~h}=1.6 \mathrm{~mm})$

From this dominant portion length, a frequency limit for the quasi-static approximation can be derived. This frequency limit is reached when the dominant line portion becomes electrically long $(\Delta L>\lambda / 10)$. Fig. 5 plots the evolution of this frequency limit of quasi-static approximation, for two substrate thickness values $(\mathrm{h}=0.42 \mathrm{~mm}$ and $1.6 \mathrm{~mm})$. Depending on the measurement height, this assumption remains acceptable from $1.5 \mathrm{GHz}$ up to $8 \mathrm{GHz}$. The frequency limits due to microstrip line dispersion are also added for two $50 \Omega$ lines, with two $h$ and $\varepsilon_{\mathrm{r}}$ values. Depending on the microstrip line characteristics, the calibration process can be limited either by the dispersion or the quasi-static approximation.

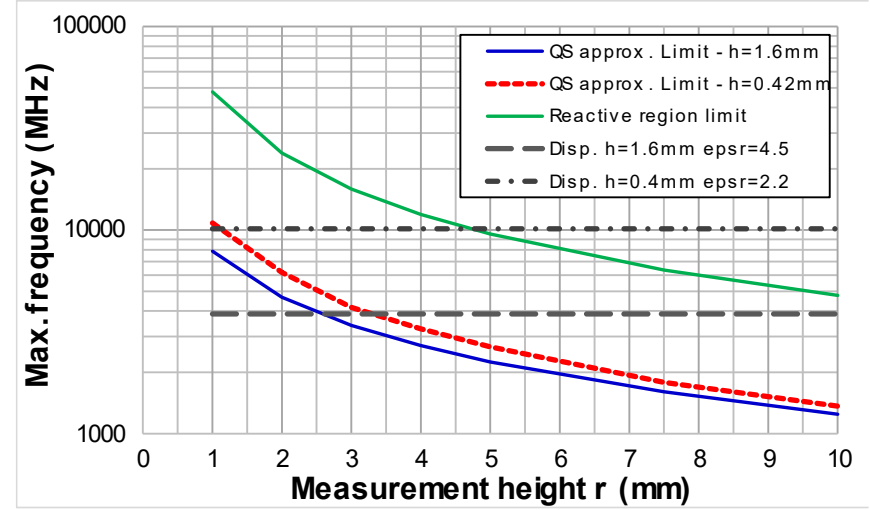

Fig. 5. Frequency limits associated to the reactive region (10), quasi-static approximation and microstrip line dispersion (4)

The validity range of the closed-form expressions based on the quasi-static approximation can be extended by a simple method, which consists in assuming that the current or voltage distribution along the dominant line portion is not constant. It does not rely into the introduction of the exact current or voltage distribution into (11) or (12), since it leads to a non-trivial integral. A correction term is proposed by considering the average current or voltage along the dominant line portion, when it is excited by a sine-wave signal and the line supposed perfectly matched. The average current $I_{\text {avg }}$ is given by (52), where $I_{0}$ is the current amplitude in $\mathrm{O}$ and $v$ the propagation speed of the line. Solving this integral gives the corrective term $C_{\text {avg }}$ in (53) which is multiply to $\mathrm{PF}_{\mathrm{H}}$ (13). The same corrective factor can be derived to account for the variation of voltage along the dominant line portion and can multiply to $\mathrm{PF}_{\mathrm{E}}$ in (38).

$$
\begin{gathered}
I_{\text {avg }}=\frac{1}{\Delta L} \int_{-\frac{\Delta L}{2}}^{\frac{\Delta L}{2}} I_{0} \cos (\gamma z) d z=I_{0} \operatorname{sinc}\left(\frac{\pi f \Delta L}{v}\right) \\
C_{\text {avg }}=\operatorname{sinc}\left(\frac{\pi f \Delta L}{v}\right)
\end{gathered}
$$

\section{COMParison With 3D ElECtromagnetic Simulation}

In order to verify the validity of the previous formulations and their frequency limits, they are compared with full-wave simulation results. Simulations are performed with FEKO software [24], based on methods of moments approach.

In a first example, the influence of the line characteristics and frequency is analyzed. A $50 \mathrm{~mm}$ long microstrip line designed on three types of substrate is considered. The first microstrip line ML1 is designed on a $1.6 \mathrm{~mm}$ thick substrate, with a relative permittivity of 4.5. The second microstrip line ML2 is designed on a $0.4 \mathrm{~mm}$ thick substrate, with a relative 
permittivity of 2.2. The third one is ML2 is designed on a 0.63 $\mathrm{mm}$ thick substrate, with a relative permittivity of 9.8 . The width of these lines is adjusted to ensure $50-\Omega$ matching $(3,1.3$ and $0.58 \mathrm{~mm}$ for ML1, ML2 and ML3 respectively). The size of the substrate is set to $60 \mathrm{~mm} \times 40 \mathrm{~mm}$. Substrate losses are introduced: the loss tangent is equal to 0.02 at $1 \mathrm{GHz}$, which is typical value for FR4 substrate. The frequency correction described in Section III.C is activated and the effect of termination vias are accounted for. The frequency evolution of the normal $\mathrm{E}$ field and tangential $\mathrm{H}$ field is computed with both methods at two different scanning heights above the center of the line: 1 and $5 \mathrm{~mm}$, up to $10 \mathrm{GHz}$. As the lines are $50-\Omega$ matched and excited by a $1-\mathrm{V}$ forward voltage, the voltage and current at the center of the line are set at $1 \mathrm{~V}$ and $20 \mathrm{~mA}$ respectively. Moreover, in the full-wave simulation, the voltage and current along the line are also captured in order to compensate any variations due to mismatch. Fig. 6 shows the comparison between the results of both approaches.

For ML1, closed-form expressions of E-field correlate to full-wave simulation results at less than $10 \%$ up to $8 \mathrm{GHz}$ and $4.5 \mathrm{GHz}$ for $\mathrm{r}=1$ and $5 \mathrm{~mm}$, while those for $\mathrm{H}$-field correlate at less than $9 \%$ up to $10 \mathrm{GHz}$ for $\mathrm{r}=1 \mathrm{~mm}$ and less than $10 \%$ up to $5.5 \mathrm{GHz}$ for $\mathrm{r}=5 \mathrm{~mm}$. For ML2, closed-form expressions of E-field correlate to full-wave simulation results at less than $3 \%$ up to $10 \mathrm{GHz}$ for $\mathrm{r}=1 \mathrm{~mm}$ and $5.5 \mathrm{GHz}$ for $\mathrm{r}=1$ and 5 $\mathrm{mm}$. Expressions for $\mathrm{H}$-field correlate at less than $2 \%$ up to 10 $\mathrm{GHz}$ for $\mathrm{r}=1 \mathrm{~mm}$ and less than $10 \%$ up to $6 \mathrm{GHz}$ for $\mathrm{r}=5 \mathrm{~mm}$. For ML3, closed-form expressions of E-field correlate to fullwave simulation results at less than $6 \%$ up to $10 \mathrm{GHz}$ for $\mathrm{r}=1$ $\mathrm{mm}$ and less than $10 \%$ up to $3 \mathrm{GHz}$ for $\mathrm{r}=5 \mathrm{~mm}$. Expressions for $\mathrm{H}$-field correlate at less than $5 \%$ up to $8 \mathrm{GHz}$ for $\mathrm{r}=1 \mathrm{~mm}$ and less than $10 \%$ up to $5.5 \mathrm{GHz}$ for $\mathrm{r}=5 \mathrm{~mm}$. The correlation
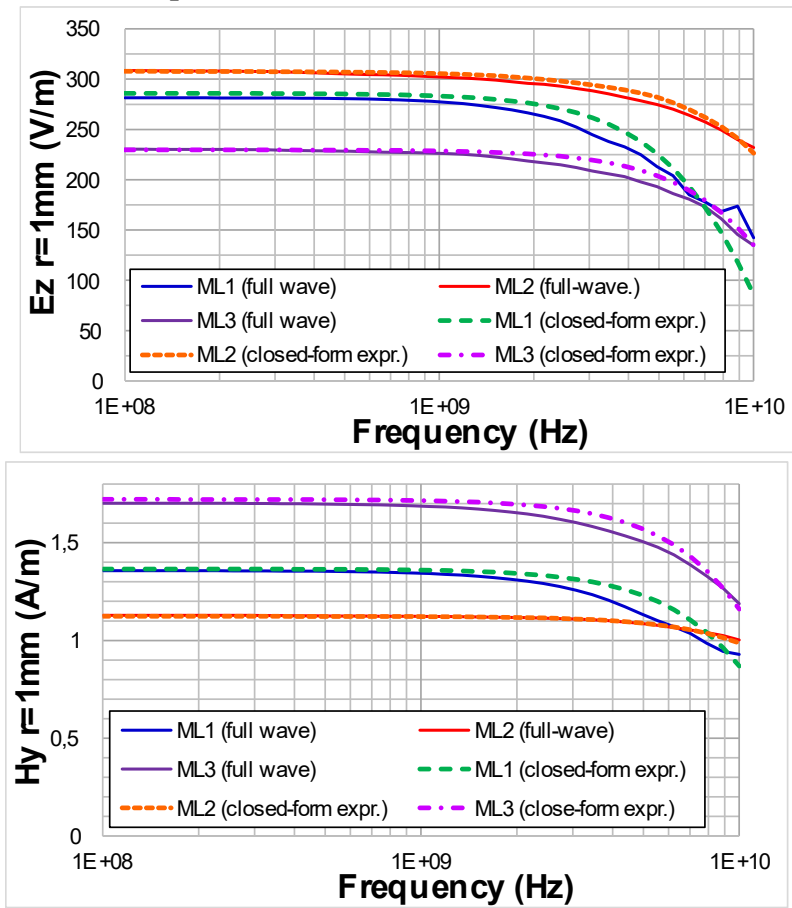

extends to higher frequencies for thinner substrate with smaller permittivity values because dispersion becomes prominent at larger frequency and line velocity increases, as expected according to (4). Thus, the quasi-static approximation remains valid up to a higher frequency.

The simulation results show that $\mathrm{E}$ and $\mathrm{H}$-fields are nearly constant at a given height up to $1-2 \mathrm{GHz}$ and the frequency correction in the closed form expressions is not required. Nearfields tend to decrease monotonously up to $5-9 \mathrm{GHz}$ depending on substrate characteristics, because of the non-uniformity of the voltage and current along the dominant portion of the line. The frequency correction reproduces this effect correctly. At higher frequency, fields tend to vary because the measurement point is close or outside the reactive region limit.

In a second example, the influence of the board edge is investigated. Here, only ML2 is considered. The evolution of the $\mathrm{E}$ and $\mathrm{H}$-field from 1 to $10 \mathrm{~mm}$ above the microstrip line is determined for various width $\mathrm{w}_{\text {sub }}$ of the substrate, from 40 to $10 \mathrm{~mm}$. The line is centered on the substrate. Full-wave simulations are done at $1 \mathrm{GHz}$ and their results compared with those of closed-form expressions in Fig. 7. The effect of board edges remains negligible when the substrate width is equal to $40 \mathrm{~mm}$, since the differences between full-wave simulation and closed-form expression do not exceed $3 \%$ and $6 \%$ for the E and $\mathrm{H}$-field respectively. When the substrate width is equal to $10 \mathrm{~mm}$, the difference for the E-field computation reaches $10 \%$ at a scanning height of $6 \mathrm{~mm}$ and $3.5 \mathrm{~mm}$ for the H-field computation. These results are valuable because they can help a designer to select the minimum dimensions of a calibration structure to limit the parasitic influence of the board edges.
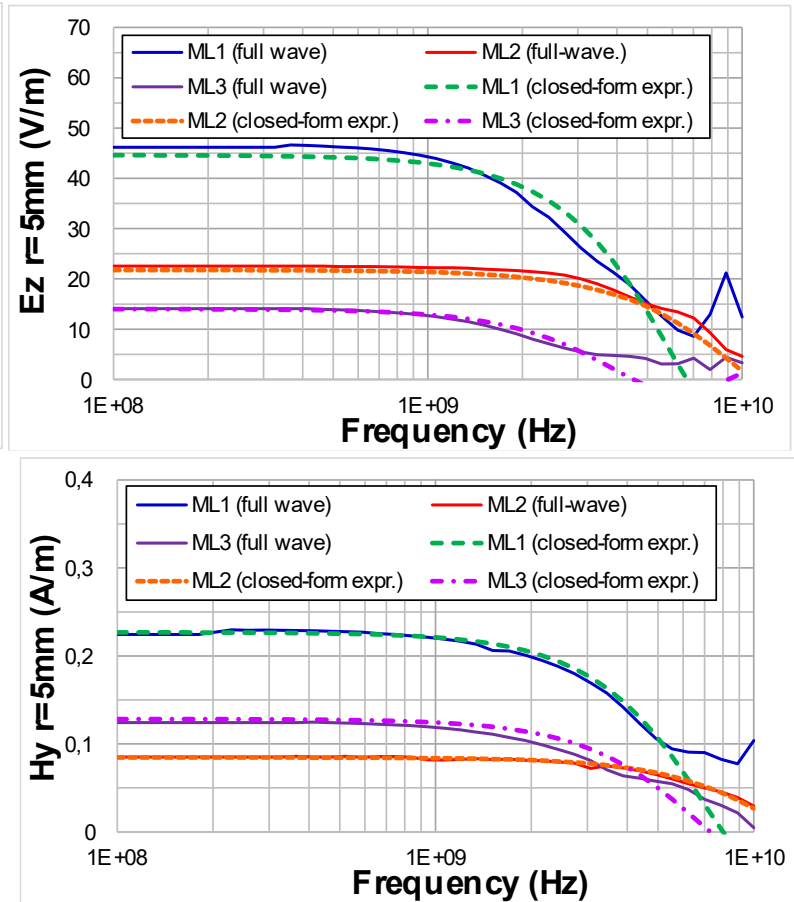

Fig. 6. Comparison of E-fields (top) and H-fields (bottom) above microstrip lines ML1, ML2 and ML3 computed by a full-wave simulation and closed-form expressions at two different height $(\mathrm{r}=1$ and $5 \mathrm{~mm}$ ) 

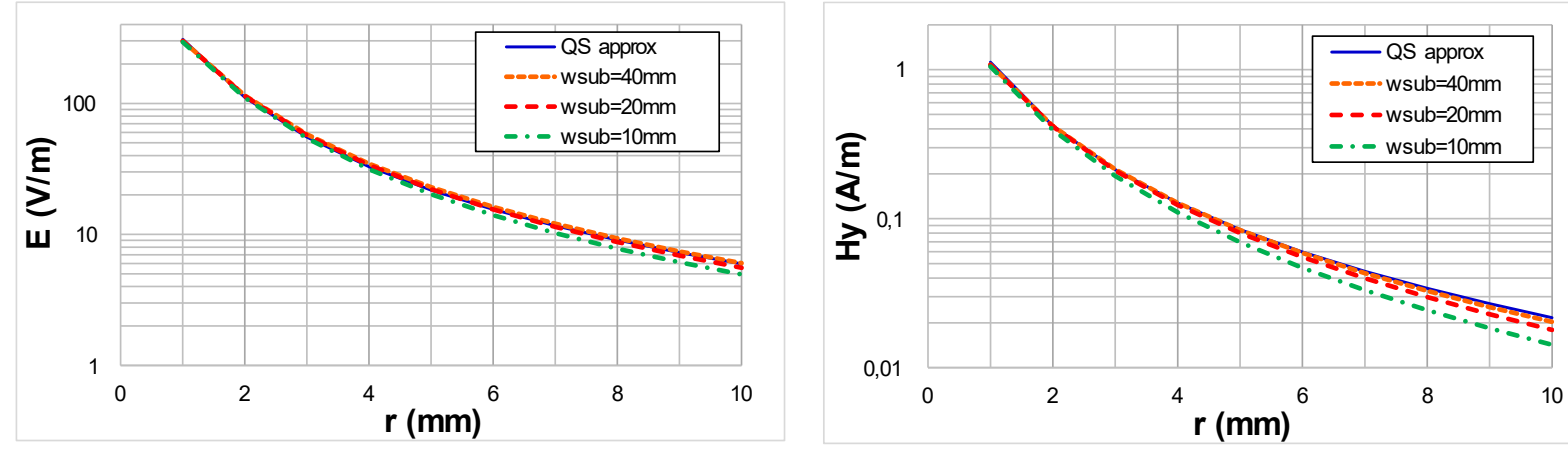

Fig. 7. Comparison of the E-field (left) and H-field (right) evolution vs. height computed according to closed-form expression and full-wave simulation with different substrate width

\section{PROBE CALIBRATION RESULTS}

This section presents the different tested probes and the calibration results: the performance factor and the effective height. PF is computed according to (1) or (2) with the adequate calibration structure model. The effective height $h_{\text {eff }}$ is adjusted in order to ensure that PF remains constant whatever the scan altitude or the calibration structure.

\section{A. Presentation of the Tested Near-Field Probes and Calibration Structures}

Several electric and magnetic field probes have been selected to validate the calibration process. They are shown in Fig. 8 and their characteristics are resumed in Table I. Probes differ according to their dimensions and constitutions, so that they exhibit different spatial resolution. Commercial probes are usually protected by a non-conductive plastic coating so that the exact geometry is not accessible. The frequency range given by the manufacturer is also added in Table I. Home-made probes are designed on a semi-rigid coaxial cable. $\mathrm{H}$-field probe heads are made by (multi-turn) loop, with or without shielding. E-field probe heads is made of an exposed short length of center conductor of a coaxial structure, terminated or not by a large electrode.

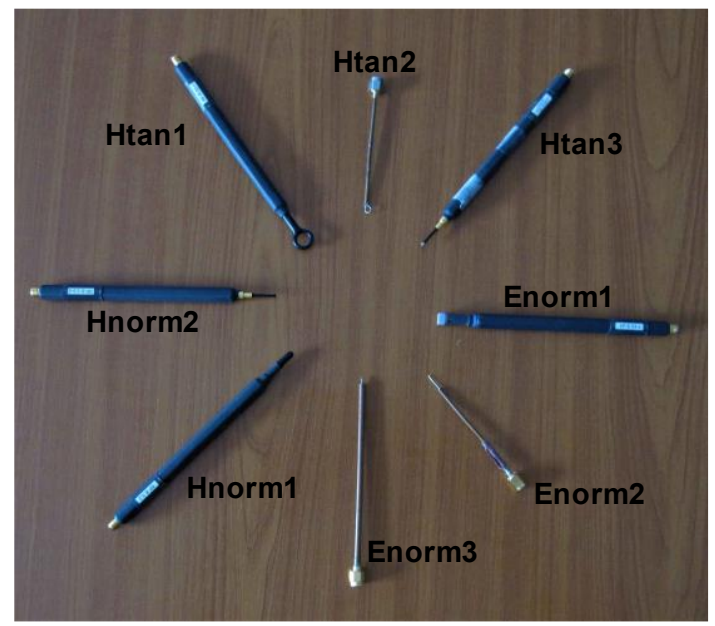

Fig. 8. Tested near-field probes
TABLE I

CHARACTERISTICS OF THE TESTED PROBES

\begin{tabular}{|c|c|c|c|}
\hline Name & $\begin{array}{l}\text { Sensed } \\
\text { field }\end{array}$ & Characteristics & $\mathrm{h}_{\text {eff }}$ \\
\hline $\mathrm{H}_{\tan 1}$ & & $\begin{array}{l}\text { Langer RFR50, } \varnothing \approx 8 \mathrm{~mm}, 1 \text { turn, } 30 \\
\mathrm{MHz}-3 \mathrm{GHz}\end{array}$ & $4 \mathrm{~mm}$ \\
\hline $\mathrm{Ht}_{\mathrm{an} 2}$ & $\begin{array}{l}\text { Tangential } \\
\text { H field }\end{array}$ & Home-made, $\varnothing=4 \mathrm{~mm}, 1$ turn & $1.8 \mathrm{~mm}$ \\
\hline $\mathrm{H}_{\tan 3}$ & & $\begin{array}{l}\text { Langer RFR0.3-3, Ø } \approx 2 \mathrm{~mm}, 2 \text { turns, } \\
\text { shielded, } 30 \mathrm{MHz}-3 \mathrm{GHz}\end{array}$ & $0.9 \mathrm{~mm}$ \\
\hline $\mathrm{H}_{\text {norml }}$ & Normal H & $\begin{array}{l}\text { Langer RFB3-2, } \varnothing \approx 3 \mathrm{~mm}, 2 \text { turns, } 30 \\
\text { MHz-3 GHz }\end{array}$ & $1.8 \mathrm{~mm}$ \\
\hline $\mathrm{H}_{\text {norm2 }}$ & field & $\begin{array}{l}\text { Langer RFB0.3-3, } \varnothing \approx 1 \mathrm{~mm}, 1 \mathrm{turn} \text {, } \\
\text { shielded, } 30 \mathrm{MHz}-3 \mathrm{GHz}\end{array}$ & $0.9 \mathrm{~mm}$ \\
\hline Enorm1 & & $\begin{array}{l}\text { Langer XFE04, } 5 \text { x } 5 \mathrm{~mm} \text { electrode tip, } \\
30 \mathrm{MHz}-6 \mathrm{GHz}\end{array}$ & $2 \mathrm{~mm}$ \\
\hline$E_{\text {norm2 }}$ & $\begin{array}{l}\text { Normal E } \\
\text { field }\end{array}$ & Home-made, tip length $=10 \mathrm{~mm}$ & $6.8 \mathrm{~mm}$ \\
\hline Enorm3 & & Home-made, tip length $=4 \mathrm{~mm}$ & $4.5 \mathrm{~mm}$ \\
\hline
\end{tabular}

Table II lists the different calibration structures used to extract $P F$ and $h_{\text {eff }}$ of the different tested probes and verify the consistency of the results. These structures differ according to their geometry, the dielectric of the substrate and the distance to the ground plane. For the H-field probe calibration, three microstrip line structures are used. Microstrip1 and 2 are long $50 \Omega$ lines designed either a HF substrate (PTFE) or a standard one (FR4), and either on a two- or four-layer board. The third microstrip is actually a short line terminated by a short circuit, that forms an elementary loop with a strong $\mathrm{H}$-field in near-field region. For E-field probe calibration, Microstrip1 structure is reused. Two other structures with high impedance termination are also introduced, which exhibit a strong E-field in near-field region: a $10 \mathrm{~mm} \times 10 \mathrm{~mm}$ square island and a PTH via with a large annular ring, both designed on a four-layer FR4 board.

Calibration measurements are made with a Rohde\&Schwarz ZVL VNA, from $100 \mathrm{kHz}$ up to $6 \mathrm{GHz}$. Probes are positioned and moved above the calibration structure by a 3 -axis scan table. The control of the altitude of the near-field probe is based on a laser telemeter. After a proper calibration of the probe position, the scan altitude is known with a good accuracy estimated at $+/-50 \mu \mathrm{m}$. 
TABLE II

DiMENSIONS AND ELECTRICAL PROPERTIES OF CALIBRATION STRUCTURES

\begin{tabular}{|c|c|c|}
\hline Name & Geometrical Dimensions & $\begin{array}{l}\text { Measured Electrical } \\
\text { Properties }\end{array}$ \\
\hline Microstrip1 & $\begin{array}{l}\text { PTFE Two-layer board } \\
\mathrm{L}=75 \mathrm{~mm}, \mathrm{~W}=3 \mathrm{~mm}, \\
\mathrm{~h}=1.6 \mathrm{~mm}\end{array}$ & $\begin{array}{l}\mathrm{Zc}=54 \Omega, \mathrm{V}_{\mathrm{P}}=186.10^{8} \mathrm{~m} / \mathrm{s} \\
\mathrm{C}_{\text {lin }}=1.14 \mathrm{nF} / \mathrm{m}, \varepsilon_{\mathrm{r}}=2.2\end{array}$ \\
\hline Microstrip2 & $\begin{array}{l}\text { FR4 Four-layer board } \\
\mathrm{L}=70 \mathrm{~mm}, \mathrm{~W}=0.75 \\
\mathrm{~mm}, \mathrm{~h}=0.41 \mathrm{~mm}\end{array}$ & $\begin{array}{l}\mathrm{Zc}=48 \Omega, \mathrm{V}_{\mathrm{P}}=161.10^{8} \mathrm{~m} / \mathrm{s} \\
\mathrm{C}_{\mathrm{lin}}=0.163 \mathrm{nF} / \mathrm{m}, \varepsilon_{\mathrm{r}}=4.5\end{array}$ \\
\hline $\begin{array}{l}\text { Elementary } \\
\text { loop }\end{array}$ & $\begin{array}{l}\text { FR4 Two-layer board } \\
\mathrm{L}=1.2 \mathrm{~mm}, \mathrm{~W}=0.2 \\
\mathrm{~mm}, \mathrm{~h}=1.6 \mathrm{~mm}\end{array}$ & $\mathrm{~L}_{\text {Loop }}=1.67 \mathrm{nH}, \varepsilon_{\mathrm{r}}=4.5$ \\
\hline $\begin{array}{l}\text { Square } \\
\text { Island }\end{array}$ & $\begin{array}{l}\text { FR4 Four-layer board } \\
\mathrm{L}=10 \mathrm{~mm}, \mathrm{~W}=10 \mathrm{~mm}, \\
\mathrm{~h}=0.41 \mathrm{~mm}\end{array}$ & $\mathrm{C}_{\text {lin }}=1.22 \mathrm{nF} / \mathrm{m}, \varepsilon_{\mathrm{r}}=4.5$ \\
\hline PTH Via & $\begin{array}{l}\text { FR4 Four-layer board } \\
\mathrm{h}=0.41 \mathrm{~mm}, \mathrm{D}=2 \mathrm{~mm}\end{array}$ & $\mathrm{C}_{\mathrm{PTH}}=0.78 \mathrm{pF}, \varepsilon_{\mathrm{r}}=4.5$ \\
\hline
\end{tabular}

\section{B. Calibration of the tested $\mathrm{H}$-field probes}

For each $\mathrm{H}$-field probe, $\mathrm{S}_{12}$ measurements were done for scan altitude $R$ ranging from 0 to $10 \mathrm{~mm}$ with a step of $0.5 \mathrm{~mm}$. For each altitude, $P F$ is extracted according to (13) and by using the appropriate model of the field produced by the calibration structure. As the actual measurement height $r$ depends on $R$ and the effective height of the probe according to (3), $h_{\text {eff }}$ is adjusted to ensure that the evolution of PF with $R$ is as constant as possible for the different test frequencies. The adjustment of $h_{\text {eff }}$ value can be done by an optimization algorithm based on $S_{12}$ measurements at all frequencies and $R$ values to minimize the variation of PF vs. R. In this work, the adjustment of $h_{\text {eff }}$ was done manually to ensure that PF remains as constant as possible between 0 and $10 \mathrm{~mm}$ for a set of measurements ranging from $10 \mathrm{MHz}$ to $6 \mathrm{GHz}$.

Once $h_{\text {eff }}$ has been set, the correct value of $P F$ can be computed. Figs. 9 and 10 present the evolution vs. frequency of the measured PF of the tested tangential and normal $\mathrm{H}$-field probes, for the same scan altitude $\mathrm{R}$ equal to $2 \mathrm{~mm}$. The extracted $h_{\text {eff }}$ values are given in Table I. A good agreement can be observed between the PF extracted with the different calibration structures up to 3 or $5 \mathrm{GHz}$, depending to the probe, proving the consistency of the calibration approach based on closed-form expressions of the $\mathrm{H}$-field. The difference between the PF measured for a same probe remains less than $2 \mathrm{~dB}$. The differences can be explained by uncertainties of the probe positioning, VNA measurement and electrical parameters of the calibration structures. Above $3 \mathrm{GHz}$, differences tend to increase, especially between the results obtained with the Microstrip2, since its substrate is more dispersive leading to a more difficult control of voltage and current along the line. Moreover, $\mathrm{H}$-field commercial probes are given up to $3 \mathrm{GHz}$. Above this frequency, E-field coupling becomes certainly non- negligible and could explain the observed differences.

These results prove that the probe characteristics ( $\mathrm{PF}$ and $h_{\text {eff }}$ ) of H-field probes do not depend on the geometrical properties of the calibration structure. Moreover, the evolution of the measured PF according to the scan altitude over microstrip2 structure is compared in Fig. 12. This result shows that PF does not vary with the scan altitude from 0 to $10 \mathrm{~mm}$. It proves that the evolution vs. height of the inhomogeneous field produced by PCB interconnects will be measured correctly with the extracted $h_{\text {eff }}$ value. These verifications to ensure a constant $h_{\text {eff }}$ are critical to ensure the practical use of these probes to sense $\mathrm{H}$-field above any electronic devices.

\section{Calibration of the tested E-field probes}

The same procedure is applied for the extraction of $h_{\text {eff }}$ and $P F$ of E-field probe, except that $P F$ is extracted according to (34). The same type of results is shown in Figs. 11 and 12 for the E-field probes. A good agreement can be observed between the PF extracted with the different calibration structures up to 5 $\mathrm{GHz}$ for $\mathrm{E}_{\text {norm1 }}$ and 2-2.5 $\mathrm{GHz}$ for $E_{\text {norm2 }}$ and $E_{\text {norm3 }}$, with difference between the measured PF less than $2 \mathrm{~dB}$. The two last probes are home-made and more sensitive to commonmode coupling, which creates important resonances above 2 $\mathrm{GHz}$. Above $5 \mathrm{GHz}$, the validity of quasi-static approximation with the correction factor (52) is not ensured anymore, so that the $\mathrm{PF}$ of $\mathrm{E}_{\text {norm1 }}$ extracted above Microstrip1 tends to diverge from the two other PF measured over smaller calibration structures. These results prove the consistency of the calibration approach based on closed-form expressions of the E-field and the effect of the substrate, at least up to $2 \mathrm{GHz}$.

The PF of E-field probe can be considered constant for scan altitude larger than 1 or $2 \mathrm{~mm}$, as shown in Fig. 12. But contrary to $\mathrm{H}$-field probe, in very close proximity of the calibration structure, $\mathrm{PF}$ tends to increase. The most likely explanation is that $h_{\text {eff }}$ is not constant in this region. The E-field coupling does not depend only on the probe head but also on the probe shielding. If its contribution rises at smaller scan altitude, $h_{\text {eff }}$ tends to increase so that the average E-field is measured at a larger height than expected. This particular effect limits the use of E-field probe with a constant PF at very close and large distance of a near-field source. The extracted $h_{\text {eff }}$ and PF is only valid for scan altitude larger than 1 or $2 \mathrm{~mm}$. For measurement only done at short distance of the source, specific PF and $h_{\text {eff }}$ values should be extracted. Once again, it is recommended to use several calibration structures, with different geometries and dielectric properties, to ensure the independence of probe characteristics to those of the structure under test. 

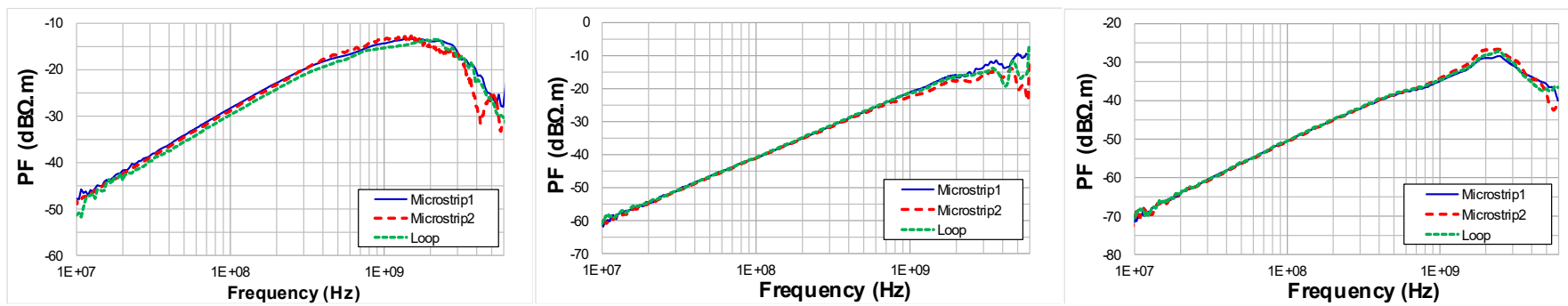

Fig. 9. Comparison between the performance factor of the tangential $\mathrm{H}$ field probes extracted from the different calibration structures (R $=2 \mathrm{~mm})$ : (left) $\mathrm{H}_{\mathrm{tan}}$, (center) $\mathrm{H}_{\text {tan2, }}$ (right) $\mathrm{H}_{\text {tan3 }}$
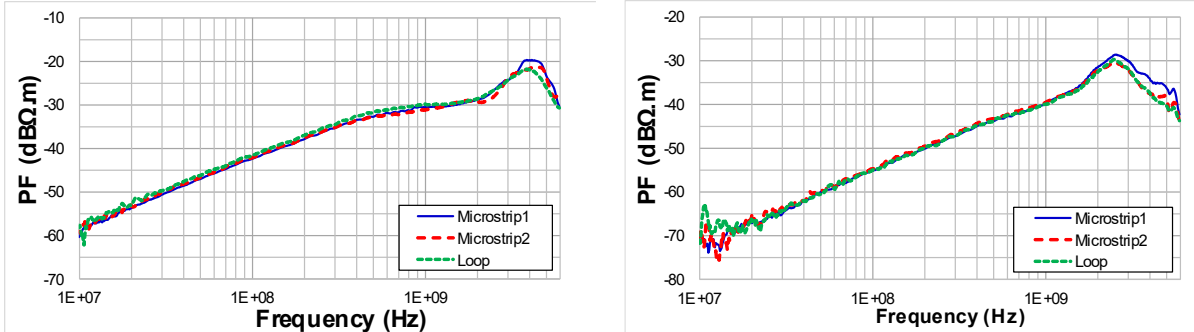

Fig. 10. Comparison between the performance factor of the normal $\mathrm{H}$ field probes extracted from the different calibration structures $(\mathrm{R}=2 \mathrm{~mm})$ : (left): $\mathrm{H}_{\text {norm }}$, (right): $\mathrm{H}_{\text {norm2 }}$
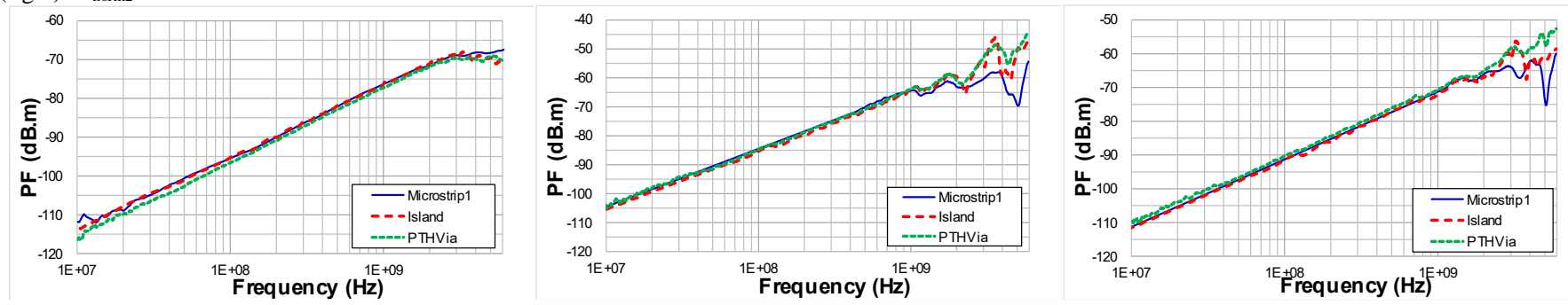

Fig. 11. Comparison between the performance factor of the normal $\mathrm{E}$ field probes extracted from the different calibration structures $(\mathrm{R}=2 \mathrm{~mm})$ : (left) $\mathrm{E}_{\mathrm{norm}}$, (center) $\mathrm{E}_{\text {norm2 }}$, (right) $\mathrm{E}_{\mathrm{norm} 3}$
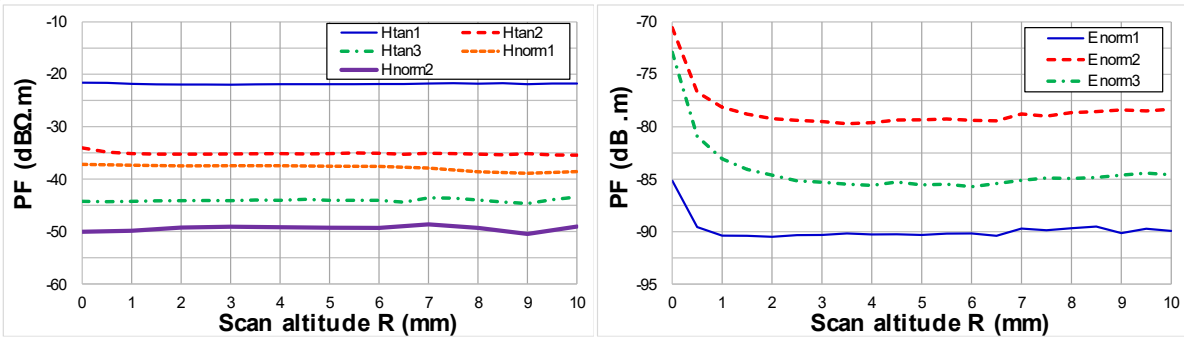

Fig. 12. Comparison between the performance factor vs. scan altitude at $200 \mathrm{MHz}$ for: (left) $\mathrm{H}$ field probe, (right) E field probe

\section{VALidATION OF THE CALIBRATION RESUlts}

In order to verify the relevance of the calibration of the tested probes, a validation structure is scanned and the reconstituted near-field maps are compared to verify their consistency. The validation structure is a Low Voltage Differential Signaling (LVDS) bus. It is routed on a four-layer board over a full ground plane, as described in Fig. 13. It is driven and terminated by a CMOS dual LVDS transceiver (TI DS90LV049 from Texas Instruments). A CMOS LVDS repeater (DS90LV004 from Texas Instruments) is inserted in the middle of the bus. The bus is $100 \Omega$ matched and excited by a $100 \mathrm{MHz}$ square signal. $\mathrm{Hx}$, $\mathrm{Hz}$ and Ez field component scans are performed at constant altitude above the board. The scan area is centered around the repeater circuit. Due to the thickness of this component, the minimal scan altitude $\mathrm{R}$ is set at $1.5 \mathrm{~mm}$, at $0.5 \mathrm{~mm}$ above the package top. The scan step size is $1 \mathrm{~mm}$ along $\mathrm{X}$ axis and $2 \mathrm{~mm}$ along $\mathrm{Y}$ axis.
To ensure a valid comparison between the results provided by the tested probes, the scan altitude $\mathrm{R}$ is adjusted to guarantee that the actual measurement height $r$ is the same, according to (3) and the $h_{\text {eff }}$ given in Table I. Measurements are done with a Rohde\&Schwarz ESPI EMI receiver $(9 \mathrm{kHz}-3 \mathrm{GHz})$ and the amplitude of the probe output is captured at the 30 first harmonics of the bus frequency.

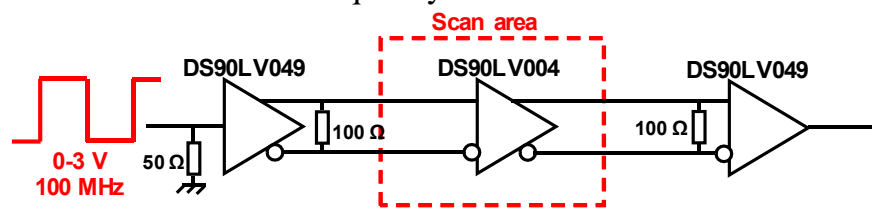

Fig.13. Electrical diagram of the studied structure: LVDS bus

In Figs. 14 to 16, Hx, $\mathrm{Hz}$ and $\mathrm{Ez}$ 2D near-field maps extracted at 600 and $800 \mathrm{MHz}$ with the different probes are compared. In spite of the importance of Hy map in a practical EMC analysis, this map is omitted for concision purpose, as the paper focuses on the relevance of the calibration process. Strong E and H-field 
levels are measured above the input and output lines of the LVDS repeater. Whatever the measured field, the maps are very similar in spite of their sensitivity differences $\left(\mathrm{H}_{\mathrm{tan} 3}\right.$ and $\mathrm{Hn}_{\text {orm2 }}$ are the less sensitive $\mathrm{H}$-field probe due to their small dimensions, $\mathrm{E}_{\tan 2}$ is the most sensitive E-field probe due to the length of its tip). The gaps between field maxima are less than $1.5 \mathrm{~dB}$. The differences can be explained by probe positioning errors and the uncertainty of the calibration process.
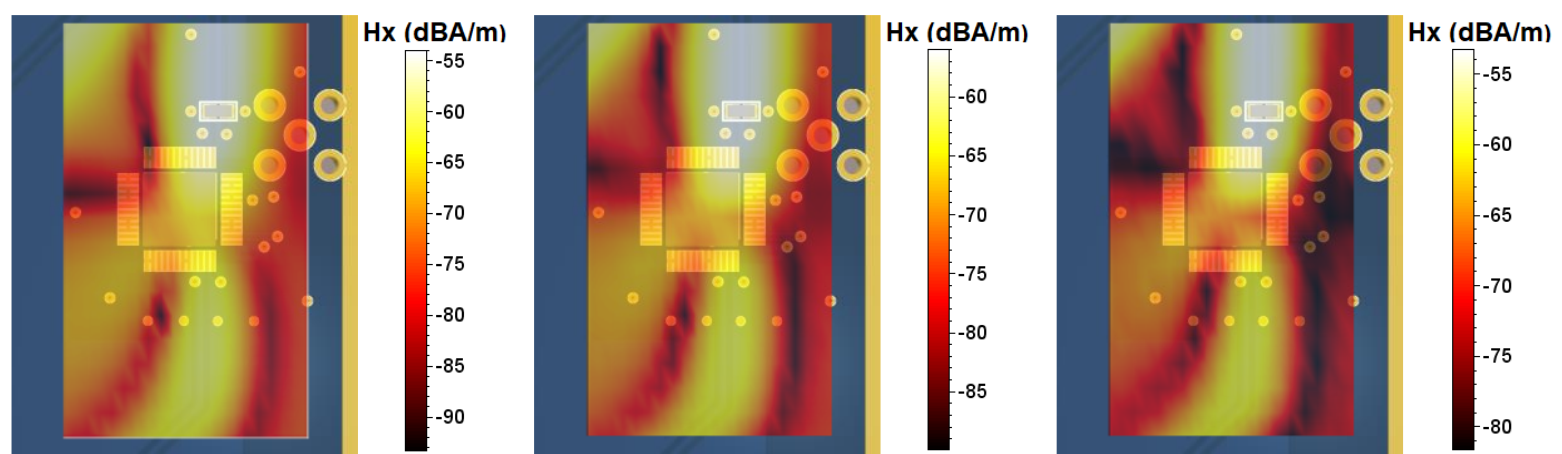

Fig. 14. Comparison between the tangential magnetic field map measured at $600 \mathrm{MHz}$ and $\mathrm{r}=5.5 \mathrm{~mm}$ above the LVDS bus with: (left) probe Htan1, (center) probe Htan2, (right) probe Htan3
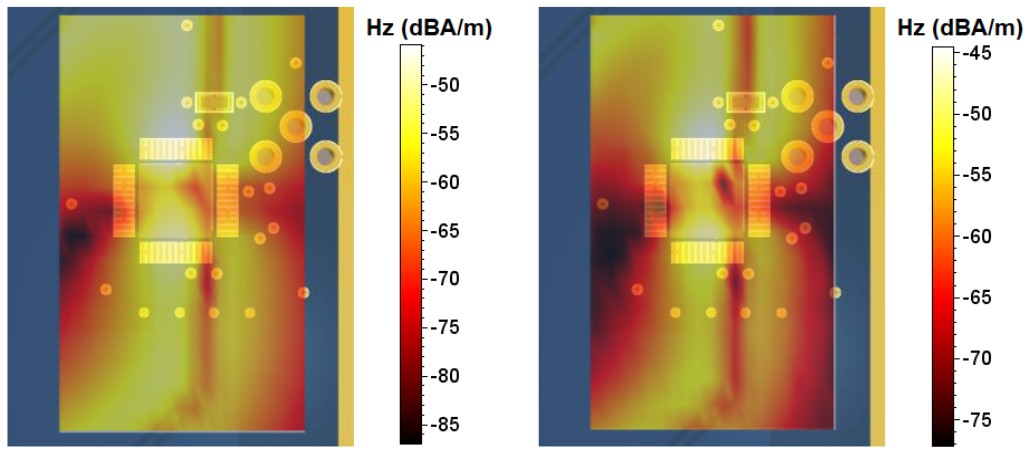

Fig. 15. Comparison between the normal magnetic field map measured at $600 \mathrm{MHz}$ and $\mathrm{r}=3.3 \mathrm{~mm}$ above the LVDS bus with: (left) probe Hnorm1, (right) probe Hnorm2
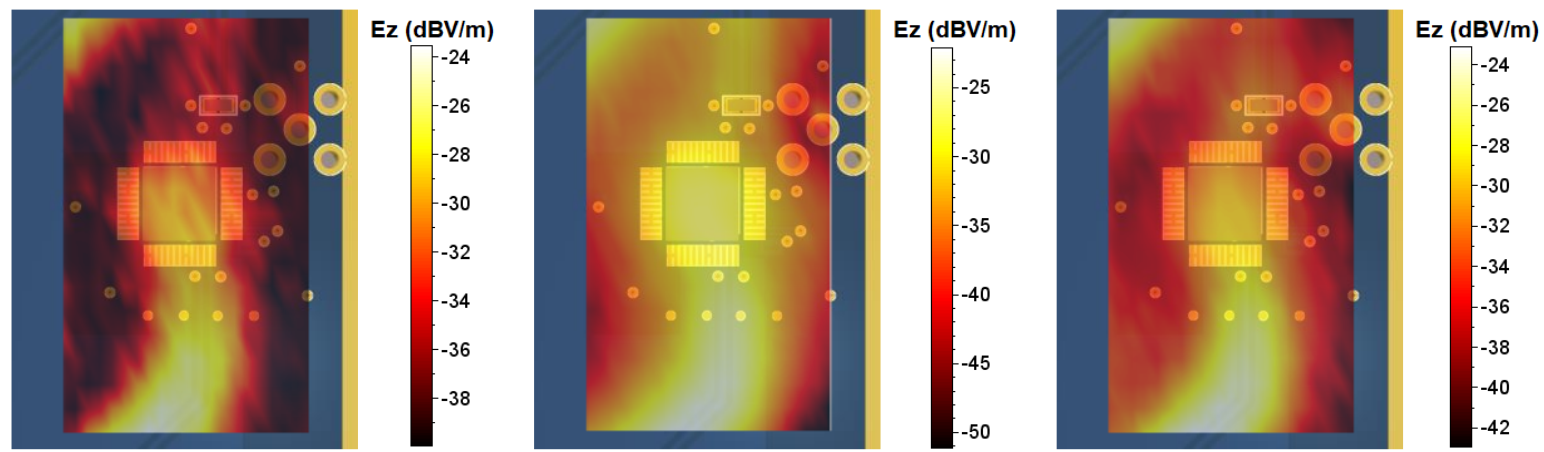

Fig. 16. Comparison between the normal electric field map measured at $800 \mathrm{MHz}$ and $\mathrm{r}=7.75 \mathrm{~mm}$ above the LVDS bus with: (left) probe Enorm1, (center) probe Enorm2, (right) probe Enorm3

Fig. 17 shows the evolution vs. frequency of the fields measured by the different probes at the same point (on a local field maximum) and the same measurement height. The noise floor level of each measurement has also been added. Gaps between noise levels result from the differences in term of PF. For $\mathrm{H}$-field measurements, the three measurements follow similar trends. Differences between the results are less than 2 $\mathrm{dB}$, except for $\mathrm{Htan} 3$ results. Above $2.5 \mathrm{GHz}$, its result differs from those of the two other probes because the signal-to-noise ratio (SNR) becomes too low. For E-field measurements, the three measurements follow similar trends and differences between the results are less than $2 \mathrm{~dB}$ up to $2.3 \mathrm{GHz}$. Differences increase at higher frequencies because of common- mode resonances and the low SNR of $\mathrm{E}_{\text {norm1 } 1}$ measurement.

These results prove the consistency between the results provided by the different probes and validate the probe calibration process based on the closed-form expressions presented in Section III, at least up to $3 \mathrm{GHz}$ for $\mathrm{H}$-field probes and $2.3 \mathrm{GHz}$ for E-field probes. These frequency limits are more related to NFP limitations than those of the closed-form expressions. 

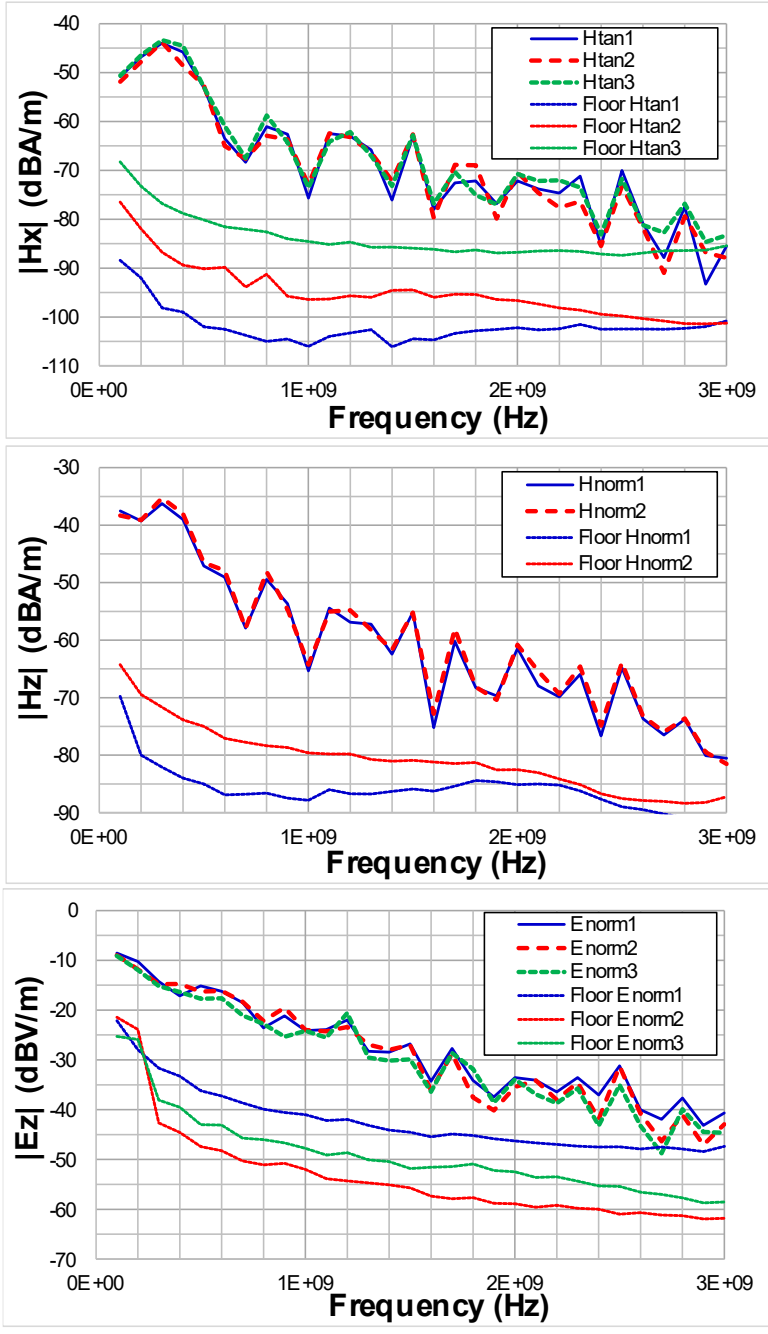

Fig. 17. Comparison of the measured fields vs. frequency with the different tested probes: (top) $\mathrm{Hx}$, (middle) $\mathrm{Hz}$, (bottom) $\mathrm{Ez}$

\section{CONCLUSION}

Near-field probe calibration process is a critical step to quantify $\mathrm{E}$ and $\mathrm{H}$-fields surrounding electronic boards and devices. The extraction of the performance factor and the effective height of a probe requires the knowledge of the field produced by a calibration structure. This paper has presented closed-form expressions based on quasi-static approximation to calculate near-field distribution from 0 to $10 \mathrm{~mm}$ over a microstrip line. It accounts for the effect of the substrate if the effect of loss can be neglected. In practice, it is recommended to design probe calibration structure on a low-loss substrate in order to limit dispersion at high frequency. The validity frequency range is given up to $1.5 \mathrm{GHz}$ up to $8 \mathrm{GHz}$, according to substrate characteristics and scan altitude. The paper has also presented a corrective term to extend this frequency range. Using such formulations avoids the recourse of complex 3D electromagnetic simulators and facilitate the calibration process. Moreover, it is a practical method to validate near-field simulation results provided by $3 \mathrm{D}$ electromagnetic simulators.

The validity of the closed-form expressions has been proved by comparison with 3D electromagnetic simulation and measurement results provided by several probes. They have been calibrated on three calibration structures, with different geometries and substrates in order to ensure the independence of probe performance factor and effective height according to calibration structure characteristics. The calibration process based on closed-form expression provides good agreements between extracted performance factor up to $2.5 \mathrm{GHz}$ and 5 $\mathrm{GHz}$, depending on the probe. They also reproduce correctly the dependence of the field to the scan altitude, which is an essential requirement to ensure that the performance factor does not change with the scan altitude. Then, these calibrated probes have been used to sense near-field over a common case study. A good agreement between the results given by the different probes confirm their validity in a calibration process, at least up to $2.4 \mathrm{GHz}$ for E-field probes and $3 \mathrm{GHz}$ for $\mathrm{H}$-field probes. These limits are mainly related to limitations of the tested nearfield probes (common-mode coupling for E-field probes, Efield coupling for $\mathrm{H}$-field probes). However, it is sufficient to cover a large number of EMC applications. Above this frequency range, as TEM propagation mode is not guaranteed on microstrip line, such a line does not constitute a convenient calibration structure. Air striplines or microwave structures should be used to overcome this issue. The proposed expressions remain valid as long as the conditions given in the paper are met.

\section{REFERENCES}

[1] J. Shi, M. A. Carcraft, J. Zhang, R. E. DuBroff, K. Slattery, M. Yamaguchi, "Using Near-Field Scanning to Predict Radiated Fields", 2004 Int. Symp. on EMC, Santa-Clara, CA, USA, Aug. 2004.

[2] N. Sivaraman, "Design of magnetic probes for near-field measurements and the development of algorithms for the prediction of EMC", Ph.D. dissertation, IMEP-LAHC, Univ. Grenoble Alpes, France, 2017.

[3] F. Caignet, N. Nolhier, M. Bafleur, "Dynamic system level ESD current measurement using magnetic field probe", 2015 Asia-Pacific Symp. on EMC, Taipei, Taiwan, May 2015.

[4] A. Boyer, S. A. Boulingui, S. Bendhia, E. Sicard, S. Baffreau, « A Methodology for predicting Disturbances due to Near Field Chip to Chip Coupling », EMC Compo 07, pp. 301 - 306, Torino, Nov. 2007.

[5] A. Boyer, B. Vrignon, M. Cavarroc, "Modeling Magnetic Near-Field Injection at Silicon Die Level", IEEE Transactions on EMC, vol. 58, no 1, pp. 257-268, Feb 2016, 10.1109/TEMC.2015.2486041.

[6] S. Criel, K. Haelvoet, L. Martens, D. De Zutter, A. Franchois, R. De Smedt, P. De Langhe, "Theoretical and experimental quantitative characterization of the near-fields of printed circuit board interconnection structures", 1995 Int. Symp. on EMC, Atlanta, GA, USA, Aug. 1995.

[7] J. Shi, M. A. Cracraft, K. P. Slattery, M. Yamaguchi, R. E. DuBroff, Calibration and Compensation of Near-Field Scan Measurements", IEEE Trans. Electromagn. Compat., vol. 47, no. 3, pp. 485-493, Aug. 2005.

[8] Z. Riah, D. Baudry, M. Kadi, A. Louis, B. Mazari, "Post-processing of electric field measurements to calibrate a near-field dipole probe", IET Sci. Meas. Technol., vol. 5, no. 2, pp. 29-36, 2011, 0.1049/iet-smt.2010.0031.

[9] H. Weng, D. G. Beetner, R. E. DuBroff, "Frequency-Domain Probe Characterization and Compensation Using Reciprocity", IEEE Trans. On EMC, vol. 53, no. 1, Feb 2011, pp. 2-10, 10.1109/TEMC.2010.2059030.

[10] T. Claeys, G. A. E. Vandenbosch, D. Pissort, "Analysis of Different Scalar Probe Compensation Methods for an Array of Near-Field EMI Probes", IEEE Trans. on EMC, vol. 63, no 2, pp. 344-352, Apr 2021, 10.1109/TEMC.2020.3008387.

[11] G. D. Sower, "Standard Calibration Method for Electromagnetic Field Probes", Sensor and Simulations Notes, Note 362, Aug. 1993.

[12] Y. Zhao, M. H. Baharuddin, C. Smartt, X. Zhao, L. Yan, C. Liu, D. W. P. Thomas, "Measurement of Near-Field Electromagnetic Emissions and 
Characterization Based on Equivalent Dipole Model in Time-Domain", IEEE Trans. on EMC, vol. 62, no 4, pp. 1237-1246, Aug 2020, 10.1109/TEMC.2019.2945560.

[13] Y. Gao, A. Lauer, Q. Ren, I. Wolff, "Calibration of Electric Coaxial NearField Probes and Applications", IEEE Trans. on Microwave Theory and Techniques, vol. 46, no. 11, Nov. 1998.

[14] A. Boyer, C. Labussière, O. Pigaglio, J. W. Tao, E. Sicard, C. Lochot, "Methodology of Calibration of Miniature Near-Field Probes for Quantitative Characterization of IC radiation”, ICONIC 2005, Barcelona, Spain, June 2005.

[15] J. Zhang, K. W. Kam, J. Min, V. V. Khilkevich, D. Pommerenke, J. Fan, "An Effective Method of Probe Calibration in Phase-Resolved Near-Field Scanning for EMI Application", IEEE Trans. Instrum. Meas., vol. 62, no. 3, pp. 648-658, Mar. 2013.

[16] S. Marathe, M. Soerensen, V. Khilkevich, D. Pommerenke, J. Min, G. Muchaidze, "Effect of Inhomogeneous Medium on Fields Above GCPW PCB for Near-Field Scanning Probe Calibration Application", IEEE Trans. on EMC, vol. 61, no 1, pp. 3-10, Feb. 2019.

[17] IEC TS 61967-3:2014, "Integrated circuits - Measurement of electromagnetic emissions - Part 3: Measurement of radiated emissions Surface scan method", Technical Specification, IEC, Aug. 2014.

[18] IEC 61967-6:2002+AMD1:2008, "Integrated circuits - Measurement of electromagnetic emissions, $150 \mathrm{kHz}$ to $1 \mathrm{GHz}$ - Part 6: Measurement of conducted emissions - Magnetic probe method", International Standard, IEC, June 2008.

[19] D. Baudry, C. Arcambal, A. Louis, B. Mazari, and P. Eudeline, "Applications of the near-field techniques in EMC investigations", IEEE Trans. Electromagn. Compat., vol. 49, no. 3, pp. 485-493, Aug. 2007.

[20] W. J. Chudobiak et al., "Dispersion in Microstrip », IEEE Trans. Microwave Theory Tech., vol. MTT-19, pp. 783-784, Sept. 1971.

[21] N. K. Das, D. M. Pozar, "A Generalized Spectral-Domain Green's Function for Multilayer Dielectric Substrates with Applications to Multilayer Transmission Lines", IEEE Trans. on Microwave Theory and Techniques, vol. MTT-35, no. 3, pp. 326 - 335, Mar 1987.

[22] G. Dural, M. I. Aksun, "Closed-Form Green's Functions for General Sources and Stratified Media", IEEE Trans. on Microwave Theory and Techniques, vol. 43, no 7, pp. 1545 - 1552, Jul. 1995.

[23] P. Silvester, "TEM wave properties of microstrip transmission lines", Proc. Inst. Elec. Eng., vol. 115, no. 1, pp.43-4S, Jan. 1968.

[24] FEKO, Electromagnetic Simulation Software, more information on www. altairengineering.fr/feko/

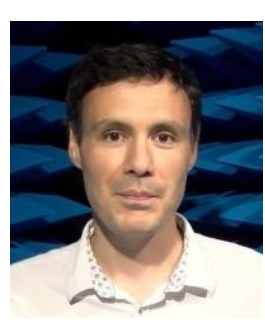

A. Boyer obtained a Masters degree in electrical engineering in 2004 and a PhD in Electronics from the Institut Nationale des Sciences Appliquées (INSA) in Toulouse, France, in 2007. He is currently an Associate Professor in the Department of Electrical and Computer Engineering at INSA, Toulouse. He is leading his research at the Laboratoire d'Analyse et d'Architecture des Systèmes (LAAS-CNRS), as part of the 'Energy and Embedded Systems' research group. His current research interests include EMC measurements, IC EMC and reliability modeling, and computer aided design (CAD) tool development for EMC (IC-EMC freeware).

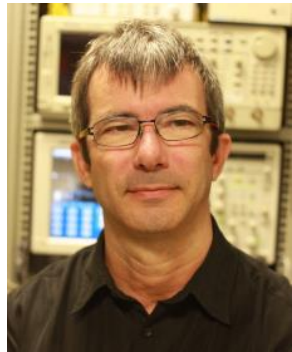

N. Nolhier received the doctorate in electronics from the National Institute of Applied Sciences (INSA), Toulouse, in 1992. He is Professor at the University Paul Sabatier, Toulouse, and joined the Laboratory of Architecture and Analysis of Systems (LAAS-CNRS). First motivated by the control and modeling of rapid heat treatment, his interests shifted to the design and simulations of Power Devices. In 1996, he initiated the study of ESD protections using 2D electrothermal simulations at LAAS and studied TLP experiments. He has supervised more than 15 Ph.D.'s in ESD protection strategy (device and system level). Since 2016, he has focused on the reliability of space nanosystems. In 2019, he became director of the Toulouse University Space Center (CSUT).

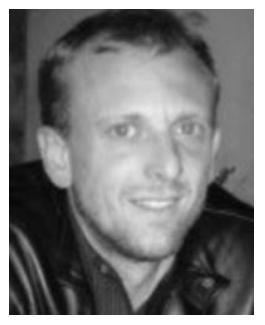

F. Caignet received the M.S. degree in electrical engineering from the University of Toulouse, Toulouse, France, in 1995, and the Ph.D. degree from the National Institute of Applied Science (INSA), Toulouse, in 1999. From 1995 to 2000, he worked on signal integrity and EMC at chip level at INSA. In 2001, he joined the Laboratory of Analysis and Architecture of Systems (LAAS) Laboratory, Toulouse, to work on the integration of optical links and devices into silicon chip tackling challenges like propagation time on high complex architectures, EMC aspect, and cost. In 2006, he joined the electrostatic discharge (ESD) team of LAAS to work on ESD at a system level. His current research interest includes developing measurement and simulation methodology to predict ESD impact at IC level.

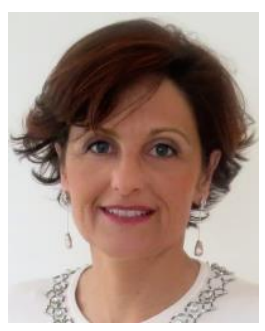

S. Ben Dhia obtained her Masters degree in electrical engineering in 1995, and a Ph.D. in Electronic Design from the Institut National des Sciences Appliquées (INSA), Toulouse, France, in 1998. Full professor at INSA-Toulouse (French engineering institute), Department of Electrical and Computer Engineering, she teaches digital electronics, IC testability and reliability, and analog and RF CMOS design. CEO of INSA Euro-Méditerranée, Fès, Morocco (2014-2017), she was responsible for the overall leadership and management of this new engineering institute. Her research interests at LAAS CNRS Toulouse include signal integrity in nano-scale CMOS ICs, electromagnetic compatibility and reliability of ICs. She has authored and co-authored 3 books, more than 120 publications in peer-reviewed journals \& conference proceedings and supervised $14 \mathrm{PhD}$ theses and $9 \mathrm{M}$.Sc. theses. 